\title{
Historische formelhafte Wendungen als Konstruktionen: Möglich- keiten und Grenzen der diachronen Konstruktionsgrammatik
}

\author{
Natalia Filatkina (Trier)
}

\begin{abstract}
Construction Grammar and research on phraseology have both commenced as purely synchronically oriented subfields of linguistics and are nowadays only starting to gain a historical dimension. Both disciplines put such units of language in the center of their research that I address in the present paper as formulaic patterns. The paper aims to discuss the possibilities and limits of multifold interactions between the two subfields of linguistics with regard to historical formulaic language. An overview of the state of the art in the field of diachronic Construction Grammar (Section 2) is followed by reflections on historical phraseology that can already partially offer some answers to the questions posed by the Construction grammar only now (cf. Section 3). The case studies (Section 4) provide an empirical basis for the preceding theoretical considerations and are based on the data of the Research Group HiFoS at the University of Trier. The data consist of about 32.000 fully annotated formulaic patterns from historical German texts since the beginning of the written tradition in the 8th century to 1650 .
\end{abstract}

It would not be entirely inappropriate to regard languages in their diachronic aspect as gigantic expression-compacting machines.

Langacker 1977: 106

Eine zusammenhängende Geschichte der deutschen Phraseologie zu schreiben, ist auch für die zweite Auflage dieses Bandes noch nicht möglich.

Burger und Linke 22008: 743

\section{$1 \quad$ Einleitung}

Bereits 1977 weist Ronald Langacker, dessen Forschung die Entstehung der Konstruktionsgrammatiken mit geprägt hat, auf den wesentlichen Anteil der Wortverbindungen in einer Sprache und ihre entscheidende Rolle im Prozess der diachronen Dynamik hin. Das erste einleitende Zitat ist ein kurz gehaltener Beleg dafür. Obwohl Langacker seine Ausführungen primär am englischen Material aufbaut, attestiert er seiner Beobachtung einen universalen Charakter. Die These über den hohen Anteil an Wortverbindungen, das Primat der Konstruktion und die Ablehnung der systemlinguistischen Grenzen zwischen Sprachebenen, insbesondere zwischen Grammatik und Lexikon, haben unterschiedliche Ausrichtungen der Konstruktionsgrammatiken von Anfang an in beinahe ähnlich hohem Maße geprägt. Die Hinwendung zur diachronen Dynamik ist hingegen ein Phänomen der neuesten Entwicklungen, das noch viele Fragen offen lässt. 
Auch die Phraseologieforschung ist eine Teildisziplin der Linguistik, die sich seit ca. 1970er Jahren systematisch mit Wortverbindungen beschäftigt. Allerdings standen auch hier bis vor kurzem vor allem moderne Sprachen im Fokus. Trotz der langen Geschichte der Phraseologieforschung konstatieren Harald Burger und Angelika Linke noch im Jahr 2008, dass eine zusammenhängende Analyse auf diesem Gebiet gegenwärtig nicht möglich sei (cf. das zweite einleitende Zitat). Die Aussage bezieht sich auf das Deutsche, aber sie gilt uneingeschränkt auch für andere Sprachen.

Trotz eines offensichtlichen Novums der diachronen Dimension für beide Forschungsrichtungen will der vorliegende Beitrag nach der Relevanz der Wechselwirkungen zwischen der historischen Konstruktionsgrammatik und der Phraseologieforschung fragen sowie die Möglichkeiten und Grenzen solcher Wechselwirkungen diskutieren. Einem Überblick über den Stand der Forschung im Bereich der diachronen Konstruktionsgrammatik in Abschnitt 2 folgen Überlegungen zu einigen Fragestellungen der historischen Phraseologieforschung, die Anknüpfungspunkte für diachron orientierte Konstruktionsgrammatiken liefern können und teilweise auch schon einige Antworten auf die Fragen bieten, die seitens der Konstruktionsgrammatik erst jetzt gestellt werden (cf. Abschnitt 3). Die Beispielanalysen in Abschnitt 4 liefern als Fallstudien eine empirische Basis für die vorangehenden theoretischen Überlegungen und stützen sich auf das Belegkorpus der Forschergruppe HiFoS („Historische formelhafte Sprache und Traditionen des Formulierens ${ }^{\text {“1 }}$ ) an der Universität Trier mit ca. 32.000 formelhaften Wendungen, ${ }^{2}$ die als Ergebnis der Auswertung historischer deutschen Texte seit Beginn der Überlieferung im 8. Jahrhundert bis ca. 1650 in Form einer relationalen Datenbank nach dem in der Gruppe entwickelten Kriterienkatalog vollständig annotiert vorliegen (Filatkina 2012 und 2018; Gottwald und Hanauska 2013; Hoff 2012).

\section{Diachrone Konstruktionsgrammatik ${ }^{3}$}

In den bereits klassisch gewordenen Standardwerken zur Konstruktionsgrammatik, die u. a. auch phraseologische Phänomene in ihren Mittelpunkt stellen, fehlen Abhandlungen zu diachronen Aspekten fast komplett. Die theoretischen Überlegungen in diesem Paradigma wurden zunächst rein ahistorisch konzipiert. Die ersten diachronen Studien erscheinen nach 2005 und diskutieren ihre Wechselwirkungen nicht mit der Phraseologieforschung, sondern mit den Theorien der Grammatikalisierung und Lexikalisierung. Dabei ist die Forschung bestrebt, sowohl die Gemeinsamkeiten und den potenziellen Nutzen konstruktionsgrammatischer Ansätze für die Grammatikalisierung/Lexikalisierung herauszuarbeiten als auch beide Theorien voneinander abzugrenzen.

\footnotetext{
${ }^{1}$ www.hifos.uni-trier.de [21.12.2017]

2 Ich verwende im Folgenden den Begriff (historische) formelhafte Wendung statt Phraseologismus, Phrasem oder Idiom und begründe meine Wahl in Abschnitt 3.

${ }^{3}$ Ich lege mich nicht auf eine bestimmte Ausprägung der Konstruktionsgrammatik fest, weil die für den vorliegenden Beitrag wichtigen Erkenntnisse allen konstruktionsgrammatischen Richtungen gemeinsam sind. Unter den diachron orientierten lasse ich lediglich die Forschungsrichtung aus, die sich mit der Anwendung konstruktionsgrammatischer Ansätze für die historisch-komparative Rekonstruktion (cf. z. B. Barđdal 2013) beschäftigt.
} 


\subsection{Gemeinsamkeiten zwischen der diachronen Konstruktionsgrammatik und der Theorie der Grammatikalisierung}

Die Gemeinsamkeiten sind offenkundig und sind bereits mehrfach benannt worden, am deutlichsten in meinen Augen in Diewald (2008a: 84-88) und Hilpert (2011: 59-61). Ich reduziere sie auf drei für meine weiteren Ausführungen wesentliche Punkte:

a) Sowohl die Grammatikalisierungsforschung als auch die Konstruktionsgrammatik verstehen das Objekt ihrer Untersuchungen zurecht sehr weit. Kay und Fillmore (1999: 1) stellen solche relatively general patterns of language wie Auxiliarverben den stärker spezifizierten idiomatic patterns gleich, wobei die letzteren die Grenzen eines Worts in beide Richtungen zugunsten der kleineren (zu Morphemen hin) wie größeren Einheiten (zu Sätzen hin) überschreiten dürfen. Systemlinguistisch geprägte Kategorien ,Wort' und ,Satz' stellen sich eigentlich als völlig irrelevant dar, weil alle Entitäten als Konstruktionen im Sinne der mental repräsentierten und deshalb abstrakten Form-/Bedeutungspaare begriffen werden (Goldberg 1995: 4).

b) Beide Forschungsrichtungen zielen auf die Untersuchung der sprachlichen Dynamik oder Variation ab und sind gebrauchsbasiert (Traugott 2003). Auch wenn in der Konstruktionsgrammatik anfänglich eher die synchrone Variation im Fokus stand, wird die Notwendigkeit der Untersuchung der diachronen Variation immer stärker hervorgehoben (Birkner 2007; Diewald 22008a, 2008b; Hilpert 2011; Smirnova 2011). Das breite Verständnis des Objektbereichs führt zwangsläufig zu einem weiteren Credo der beiden Theorien: Sie gehen grundsätzlich davon aus, dass sich Veränderungen und Variation auf mehreren Ebenen gleichzeitig, ebenenübergreifend und konstruktionsübergreifend abspielen. Am deutlichsten kam diese Idee bereits in Goldbergs Konzept der Vererbung (inheritance; 1995: 67100) zum Ausdruck, in dem Realisierungen einer Generalisierung als ein Netzwerk von verwandten Konstrukten (Varianten) verstanden wurden. Damit ist gleichzeitig einer der Faktoren benannt, der auch in der historischen Linguistik im Allgemeinen und in der Grammatikalisierung im Besonderen als eine der treibenden Kräfte des Sprachwandels betrachtet wird und der Analogie nahe kommt.

c) Das Ziel der Grammatikalisierungsforschung und der diachronen Konstruktionsgrammatik besteht nicht nur in der Beschreibung der einzelnen Veränderungsschritte, sondern in der Erklärung der Variation und der Erstellung der formalisierten Modelle der Veränderungswege. Die Grammatikalisierungsforschung versucht dieses Ziel mit Hilfe der sogenannten Grammatikalisierungspfade zu erreichen, die Konstruktionsgrammatiker mit Hilfe der Generalisierungen. Während die Grammatikalisierungsforschung von Beginn an und kontinuierlich an der detaillierten Untersuchung der einzelnen Veränderungsschritte orientiert war, überwog in den Konstruktionsgrammatiken das Bestreben, abstrakte Generalisierungen über mental präsente Form-/Bedeutungspaarungen vorzunehmen. Ausgerechnet den diachron orientierten konstruktionsgrammatischen Studien ist zu verdanken, dass sich das Interesse immer mehr von bloßen Generalisierungen auf die Untersuchung der Veränderungen auf der Ebene der einzelnen Konstrukte, der Prozesshaftigkeit und der Details der Entwicklung verlagert (cf. den Begriff „resulting layering effects“ (Fried 2013: 421) oder die Unterscheidung zwischen Makro-, Meso- und Mikrokonstruktionen (,types, as distinct from actual utterances“ (Traugott 2008: 32)), die alle Abstraktionen unterschiedlichen Gra- 
des sind und sich ihrerseits von Konstrukten unterscheiden ${ }^{4}$ ). In Anlehnung an ,grammaticalization“ wurde der Begriff „constructionalization“ entwickelt, obwohl er in Fried (2013: 424) und Hilpert (2013: 460) als ein der Grammatikalisierung übergeordneter Terminus verstanden wird: Grammatikalisierungsprozesse seien Etappen der sogenannten Konstruktionalisierung. In diesem Zusammenhang wird eine weitere treibende $\mathrm{Kraft}^{5} \mathrm{der}$ sprachlichen Veränderungen in die konstruktionsgrammatische Diskussion eingebracht, nämlich die Rolle des unmittelbaren Ko-textes (internal properties) und des erweiterten Kontextes (external properties). Die Grammatikalisierungsforschung war wie auch z. B. Bedeutungswandeltheorien schon immer kontextorientiert, ${ }^{6}$ Fried (2013: 428) hebt die Wichtigkeit dessen auch für die Konstruktionsgrammatik hervor. Diese Verlagerung des konstruktionsgrammatischen Forschungsinteresses von Generalisierungen auf die Prozesshaftigkeit der Entwicklungen fordert aber in meinen Augen eines der zentralen theoretischen Postulate der Konstruktionsgrammatiken heraus, nämlich das Verständnis der linguistischen Strukturen als holistische und arbiträre Form-/Bedeutung-Korrespondenzen, bei denen sich folglich Form und Bedeutung simultan verändern sollten. Diachron zeigt es sich, dass Gleichzeitigkeit nur auf der Ebene der abstrakten Konstruktionen festgestellt werden kann, die vorausgehenden Etappen (nach Fried (2013: 436) „feature-based level“) sind davon nicht betroffen. Das wurde in Filatkina (2015) auch für die Variation im Bereich der formelhaften Wendungen gezeigt.

\subsection{Unterschiede zwischen der diachronen Konstruktionsgrammatik und der Theorie der Grammatikalisierung}

Die in Abschnitt 1.1 aufgezeigten Gemeinsamkeiten machen bereits deutlich, dass sich beide Forschungsrichtungen nicht nur mit Variation beschäftigen, sondern auch mit Phänomenen des Sprachwandels. Paradoxerweise sind in diesem Bereich am stärksten die Bestrebungen spürbar, beide Forschungsrichtungen auseinanderzuhalten. Mit dem Begriff „Konstruktionswandel“ (constructional change, construction-specific change) wird zwar weder der Anspruch erhoben, den kompletten Sprachwandel grundsätzlich als Konstruktionswandel zu begreifen; noch soll die Richtigkeit der Analyse der sprachlichen Veränderungen im Grammatikalisierungsparadigma in Frage gestellt werden (Noël 2007). Es geht eher darum, eine zusätzliche Analysekategorie für solche Sprachwandelprozesse einzuführen, für die sie in der Tat zielführend sein könnte und die vor allem nicht systemischer Art sind. Hilpert (2011: 70f.) beweist dies am Beispiel der englischen Quantorenkonstruktion mit der Form many + NP (many a day, many a Republican, many a critical moment) ${ }^{7}$ : Während sich seit 1820 auf der Ebene der Form kein erkennbarer Wandel feststellen lässt, zeigt eine quantitative Frequenzanalyse der substantivischen Kollokate eine Veränderung in ihren lexikalischen Präferenzen von emotional aufgeladenen Lexemen (many a heart, many a tear) über Zeiteinheiten (many a day, many a year) hin

\footnotetext{
${ }^{4}$ Vergleichbare Überlegungen finden sich aber auch schon in Croft (2001); Diewald und Habermann (2005) behandeln ausführlich die ko- (z. B. Erweiterung der Valenz) und kontextuellen (etwa den Einfluss der rhetorischen Traditionen) Bedingungen bei der Entstehung des analytischen Futurs im Deutschen. Cf. auch Weber (2015) zum Einfluss der Diskurstraditionen und des Sprachkontakts auf die notwithstanding-Konstruktion im älteren Englisch. ${ }^{5}$ Unter b) wurde bereits auf die Rolle der Analogie hingewiesen.

${ }^{6}$ Cf. auch die Ausführungen zu untypischen, kritischen und isolierenden Kontexten in Diewald ( $\left.{ }^{2} 2008 \mathrm{a}: 80-84\right)$.

${ }^{7}$ Cf. auch den Begriff collocational change im Sinne des Wandels der Kombinationsprofile (nicht nur bei spezifischen Konstruktionen wie Idiomen) in Hilpert (2013: 472f.).
}

ISSN 1615-3014 
$\mathrm{zu}$ Bezeichnungen von menschlichen Individuen in sozial definierten Rollen (many a reader, many a citizen). Die Kookkurrenzen mit neuen lexikalischen Elementen interpretiert Hilpert als Indizien für eine semantische Erweiterung auf der Basis der Analogie. In der Terminologie der klassischen Phraseologieforschung würde man solche Einheiten als Modellbildungen bezeichnen. In meinen Augen eignet sich der Begriff „Konstruktionswandel“ gerade für solche Fälle gut, weil a) von Veränderungen hier nicht einzelne Wörter betroffen sind, sondern ganze (kompositionelle oder nichtkompositionelle) syntagmatische Strukturen; ${ }^{8}$ b) sich die Veränderungen auf mehreren Ebenen gleichzeitig abspielen und die Zuordnung zu gängigen Theorien des Sprachwandels (z. B. des Bedeutungswandels, des lexikalischen Wandels usw.) nicht zielführend ist; c) ihre Endprodukte keine grammatischen Zeichen sind und die aus der Forschung zur Grammatikalisierung bekannten Prozesse nicht greifen und d) es im Bereich der formelhaften Wendungen einen systemischen Wandel (z. B. Variation, Systemverschiebungen) als Reaktion auf das durch Schwund entstandene Vakuum nicht geben kann. Ich komme in Abschnitt 4 darauf noch zurück. Konstruktionswandel wird vorläufig folgendermaßen definiert: Konstruktionswandel erfasst selektiv ein konventionalisiertes Form-Bedeutungs-Paar einer Sprache und verändert es in seiner Form, seiner Bedeutung, seiner Frequenz, seiner Verteilung in der Sprechergemeinschaft oder in einer beliebigen Kombination dieser Aspekte" (Hilpert 2011: 69). ${ }^{9}$

Damit sind auch die Ebenen genannt, die vom Konstruktionswandel betroffen sein können. Dass diese Auflistung nicht vollständig sein kann, beweisen die Ergebnisse der traditionsreichen (systemlinguistischen, aber auch kulturhistorisch orientierten) Sprachgeschichte, Variations- und Soziolinguistik. Sprachhistorisch gesehen und auf die historische formelhafte Sprache bezogen, erweisen sich insbesondere die Frequenzveränderungen als problematisch, einfach weil ihre Dokumentation und Untersuchung durch die Gegebenheiten (der oft zufällig erhaltenen) schriftlichen Überlieferung eingeschränkt sind. Die Rolle der Frequenz im Prozess der Habitualisierung wird auch seitens der Grammatikalisierung und Lexikalisierung angezweifelt. Während die früheren Arbeiten darauf bestanden (Lehmann 1989, 1995; Talmy 1985), unterscheiden die jüngeren zwischen Frequenz und Wiederholung (z. B. Bybee 2010: 34). Die wiederholte Verwendung in bestimmten Kontexten in der entstehenden reduzierten Bedeutung (und Form) ist für die Konventionalisierung ausschlaggebend, (hoch)frequentes Vorkommen begleitet hingegen nur einige emergente Sprachzeichen, am häufigsten hochabstrakte grammatische Elemente. Laut Bybee (2010: 220) basieren sprachliche Veränderungen nicht auf Frequenz, sondern auf kognitiven Basisprozessen wie categorization by similarity, chunking of repeated sequences und association by contiguity. Chunking ist dabei vor allem für die Bildung von komplexen polylexikalischen Einheiten wichtig (Bybee 2002) und besteht in der Fähigkeit des menschlichen Gedächtnisses, polylexikalische Sequenzen zu speichern, sich an bereits bestehende Kombinationen zu erinnern und diese ausgehend von den sich verändernden Bedingungen des Sprachgebrauchs neu zu organisieren. Mit dem letzten Element der Neuorganisation wird im permanenten Prozess der Entstehung der sprachlichen Struktur neben Konventionalisierung auch Variation ein gebührender Platz eingeräumt.

\footnotetext{
${ }^{8}$ Bergs und Diewald (2008: 3) heben diesen Aspekt hervor.

9 Ähnlich auch Hilpert (2013: 460; 2014: 196).
} 
Ähnliche Überlegungen in Bezug auf die Frequenz liefert die Textlinguistik. So ist es laut Feilke (2004: 41) aus Sicht der Textproduktion irrelevant, wie häufig ein Ausdruck vorkommt. Selbst das rekurrente Produzieren und Rezipieren eines Ausdrucks garantiert nicht, dass die Sprecherinnen und Sprecher ihn als konventionelles kommunikatives Zeichen bewerten und beherrschen würden. Umgekehrt gibt es etwa in fachspezifischen Kontexten Ausdrücke niedriger Rekurrenz, die aber in diesen Kontexten formelhaft (idiomatisch geprägt in Feilkes Terminologie) sind. Wray (2009: 36), Wray und Perkins (2000: 7) u. a. betonen dies auch aus der Perspektive der Spracherwerbsforschung. Zuletzt hat auch Steyer (2013: 31 und 341) aus korpuslinguistischer Sicht behauptet, dass die statistische Signifikanz des Kovorkommens von Komponenten einer potenziellen Wortverbindung keine absolute Größe ist, lediglich in Bezug auf eine bestimmte Fragestellung Sinn ergibt und vor dem Hintergrund des zugrunde gelegten Untersuchungsdesigns zu bewerten ist.

\subsection{Zwischenfazit}

Aus dem in den Abschnitten 1.1 und 1.2 Gesagten lässt sich als ein Zwischenfazit festhalten, dass der entscheidende Vorteil des diachronen konstruktionsgrammatischen Herangehens in meinen Augen in der konsequenten Orientierung am simultanen und ebenenübergreifenden Charakter der Veränderungen bestehen kann. Der zweite Vorteil, den die Beschreibung einiger Sprachwandelprozesse als Konstruktionswandel mit sich bringt, besteht in der Möglichkeit, Veränderungen mit nicht systemischem Charakter zu interpretieren, $d$. h. solche Veränderungen, die nicht oder nicht unbedingt weiterführende Veränderungen im System/Paradigma nach sich ziehen und ins ,theoretische Niemandsland“ (Hilpert 2011: 71) zwischen den bekannten Sprachwandelprozessen fallen. Genau zu dieser Gruppe würden Untersuchungen zu diachronen Veränderungen von Beispielen wie let alone, what's X doing $Y$, the Xer the Yer u. a. gehören, die synchron bereits als klassische Beispiele für Konstruktionen gelten können und diachron noch nicht erforscht sind.

Trotz der immer deutlicher werdenden Hinwendung zur Diachronie bleiben noch viele Fragen offen, u. a. die Fragen nach dem Grad der Variation oder weiteren Faktoren/Ursachen/treibenden Kräfte des Konstruktionswandels in Ergänzung zu den in der Forschung bereits erwähnten Analogie, Reanalyse, Frequenz und teilweise Expressivität (einschließlich metaphorischer und metonymischer Erweiterungen). Fried (2009b: 289) stellt am Beispiel der Konstruktion jestli ,wenn', vielleicht‘ im gesprochenen Tschechisch z. B. fest, dass für die Verfestigung der Propositionalausdrücke nicht einfach die Kenntnis der syntaktischen Profile und der Wortbedeutungen ausreicht, sondern das Metawissen der Sprecherinnen und Sprecher über die relevanten Diskurskonventionen (discourse patterns) und die Textorganisation (reference to principles of textual organisation).

Während die synchron orientierten konstruktionsgrammatischen Studien formelhafte Wendungen in ihren Mittelpunkt gestellt haben und nach wie vor stellen (cf. exemplarisch Birkner 2008; Dobrovol'skij 2010, 2011; Dobrovol'skij und Šarandin 2010; Feilke 2007: 71-7410), sind sie kaum Gegenstand diachroner Untersuchungen. Schwerpunktmäßig werden

\footnotetext{
10 Insbesondere Dobrovol'skij (2011: 114, 2010) und Dobrovol'skij/Šarandin (2010) haben nachgewiesen, dass
} der Typ phraseologischer Wendungen, den sich „Phrasem-Konstruktionen (PhK)“ nennen (z. B. Es ist zum Ver-

ISSN 1615-3014 
hier abstrakte grammatische Phänomene behandelt, vermutlich weil diachrone Konstruktionsgrammatiken ihren Gegenstand verstärkt in Abgrenzung zur Theorie der Grammatikalisierung $\mathrm{zu}$ definieren versuchen. Es liegen Untersuchungen zu Modalverben und Modalpartikeln, zur Verbzweitstellung und zum analytischen Futur, zum am-Progressiv und Komplementkonstruktionen, Kasussynkretismus und -variation ${ }^{11}$ oder zu Relativsatz-Konstruktionen vor - alles Beispiele, die auch im Kern der Grammatikalisierungsforschung stehen. Eine Ausnahme stellt die Untersuchung von Östman und Trousdale (2013: 484-490) zu Wellerismen des Typs „, It all comes back to me now", said the captain, as he spat into the wind dar. Den wichtigsten Unterschied zu den im Rahmen des Konstruktionswandels bereits betrachteten Phänomenen sehen die Autoren bei Wellerismen in der Tatsache liegen, dass die bloße Beschreibung ihrer inneren Struktur etwa mit Hilfe der grammatischen Kategorien nicht ausreicht und um die Analyse der Gebrauchsbesonderheiten im Diskurs erweitert werden muss. Die letzteren sind nicht als externe Kontexte zu betrachten, sondern als Teil der inneren Struktur, die aktiv auf die Verwendung der Wellerismen einwirken:

A number of traditional Construction Grammar features may also need to be re-evaluated when taken into the discourse realm: constructions (as abstract generalizations over instances of usage) are maybe not merely form-meaning-pairs, but tripartite form-meaning-function constellations; when the crucial Construction Grammar distinction between internal and external features is taken beyond the sentence into discourse, it is not clear - albeit that the distinction is a recursive relation - what is 'internal' anymore; the idea that not all attributes have to receive values (which is a sine qua non for any usage-based construction grammatical analysis) will have to be given a more prominent place: the nonassignment of a value is not only a lack of specification but also the result of a communicative choice; and, finally, traditional 'constituency' may have to be properly re-evaluated (by being socially, interactively, cognitively, and culturally informed) so as to allow analyses to go against the very basics of constituency in traditional grammar.

(Östman und Trousdale 2013: 486-487)

In a discourse approach to constructions, context features [...] are not outside of constructions, but part of the constructions. Together with the internal features, they specify resources for language users in an ordinary constructional fashion. When looked at in this manner, 'contextual features' that affect variability are not seen as being outside grammar, but as being part of grammar.

(Östman und Trousdale 2013: 488) $)^{12}$

rücktwerden!, Was du nicht alles gelesen hast!, Freund hin, Freund her, vor sich hin, vor sich her usw.), konstruktionsgrammatisch besser als phraseologisch beschrieben werden kann, weil solchen Phraseologismen einerseits ein festes syntaktisches Pattern mit einer eigenständigen Bedeutung zugrunde liegt, die lexikalischen Slots aber andererseits relativ frei besetzbar sind. Sind prototypische Phraseologismen wie Idiome und Sprichwörter nicht vollständig lexikalisch spezifiziert und führt ihre kontextuelle Einbettung zu deutlichen semantisch-pragmatischen Verschiebungen (Das ist nicht mein Bier vs. Das ist nicht sein/dein Bier!), eignen sie sich auch für die Analyse im konstruktionsgrammatischen Paradigma. Aber auch schon von Polenz (1963), Eisenberg ( $\left.{ }^{2} 1989\right)$ und Burger (1987) heben den Aspekt der seriellen Produktivität bei Funktionsverbgefügen (zur Entscheidung kommen/bringen/stehen; unter Kontrolle kommen/bringen/stehen) und damit das diese Einheiten als Konstruktionen charakterisierende Merkmal hervor. Rothkegel (1989) wirft die Fragen der Modellierbarkeit konventioneller Kollokationen (Stoff liefern, Neugier erregen, (k)einen Sinn ergeben, Maßnahmen ergreifen) für Zwecke einer maschinellen Übersetzung und betont die ,relative Fixierung des syntaktischen Musters und auf bestimmte Kontexte eingeschränkte Bedeutung der Teilkonstituenten“ (Rothkegel 1989: 17), was sie zum Schluss führt, dass „die Fixierung der syntaktischen Konfiguration Bedingung für den Bedeutungsaufbau“ (Rothkegel 1989: 18) ist. Zu Gemeinsamkeiten und Unterschieden zwischen der Phraseologieforschung und der Konstruktionsgrammatik auf der Ebene der Terminologie cf. Filatkina (-2018).

11 Dazu neulich Wolk, Bresnan, Rosenbach und Szmrecsanyi (2013).

12 Cf. auch schon das Modell der symbolischen Struktur einer Konstruktion in Radical Construction Grammar (Croft 2001: 18).

ISSN 1615-3014 
In Abschnitt 4 werde ich diese These am Material der historischen formelhaften Wendungen diskutieren. Ich werde die oben gestellten Fragen nicht vollständig beantworten können; einen Beitrag für weitere Überlegungen soll die Beispielanalyse hoffentlich dennoch liefern.

\section{Historische Phraseologieforschung und diachrone Konstruktionsgrammatik}

Obwohl die Abhandlungen zur Diachronie der formelhaften Sprache in den als Referenzwerke geltenden Einführungen und Lehrwerken zur Sprachgeschichte, Variation und/oder zum Sprachwandel fehlen, ${ }^{13}$ sind sie seit langem Gegenstand sprachhistorischer Einzeluntersuchungen, auch mit monographischem Charakter. Sie entstammen dem Bereich der historisch orientierten Phraseologie- und Sprichwortforschung und definieren formelhafte Wendungen als Phraseologismen im Sinne Burgers $\left({ }^{4} 2010,33-57\right)$. Die Fragen nach der Variation und der Entstehung der Wendungen beschäftigen seit langem die klassische Phraseologieforschung. Auch wenn sie für diese nicht immer zentral waren, zeichnen sich doch gegenwärtig einige Erkenntnisse $a b$, die auch für die diachrone Ausrichtung der Konstruktionsgrammatiken von Relevanz sein können. Ich stelle diese Erkenntnisse der diachronen Phraseologieforschung den in Abschnitt 1 für die diachrone Konstruktionsgrammatik dargestellten Thesen a), b) und c) gegenüber.

$\mathrm{Zu}$ a) Die Breite der Definition des Untersuchungsgegenstandes erweist sich auch für die Erforschung der historischen formelhaften Sprache als ein fruchtbarer Ansatz, vor allem weil sie auch das wenig Frequente bzw. das (noch) Nicht-Produktive berücksichtigt. Mit Blick auf ihre token-Frequenz ${ }^{14}$ können formelhafte Wendungen solch wenig frequente Einheiten sein. Die mentale Repräsentanz ist hingegen ein Merkmal, das sprachhistorisch nicht greifbar ist, weil der Untersuchungsgegenstand nur mittelbar durch den Filter der schriftlichen Textüberlieferung und nicht etwa durch die Auseinandersetzung mit Sprachträgerinnen und Sprachträgern zugänglich ist.

Zu b) Formelhafte Sprache ist genauso wie Phonetik, Morphologie, Lexik, Syntax usw. von der Variation und dem ständigen Wandel betroffen. ${ }^{15}$ Dieser findet bei Wendungen simultan auf mehreren Ebenen statt und macht diese sprachlichen Einheiten für moderne Sprachwandeltheorien relevant. Ich habe dies an anderen Stellen (Filatkina 2013, 2015) anhand der diachronen Entwicklung des Idioms Perlen vor die Säue werfen gezeigt. Bedingt ist der ebenenübergreifende Charakter der Variation und des Wandels dadurch, dass formelhafte Wendungen oft Phänomene sind, die nicht einer sprachlichen Ebene zugeordnet werden können, sondern sich zwischen Lexik, Grammatik, Pragmatik und Diskurs bewegen.

\footnotetext{
13 Dazu ausführlich Filatkina (2018).

14 Die token-Frequenz gestaltet sich im Bereich der Formelhaftigkeit anders als die type-Frequenz: Mit Blick auf die type-Frequenz kann absolut jeder Text als formelhaft betrachtet werden, weil Formelhaftigkeit ein Grundkonstituens der menschlichen verbalen Kommunikation ist. Allerdings verfügt jeder type im Normal- und Regelfall über eine niedrige token-Frequenz.

15 Cf. für das Englische - Aurich 2012, 2013; für das Deutsche - Burger und Linke ${ }^{2} 2008$, 746-753; Dräger 2011; Filatkina 2013, 2015; Friedrich 2007: 1094-1103; Stumpf 2014; für das Russische und andere slawische Sprachen - Mokienko 2002; Bierich 2005; für das Französische - Buridant 2007 und für das Luxemburgische - Filatkina 2005; Kleine-Engel 2012, 2011.
} 
Mit Blick auf die ausgeprägte Variation wurden sprachliche Einheiten, die die HiFoS-Gruppe zu ihrem Untersuchungsgegenstand hatte, als „formelhafte Wendungen“ bezeichnet. Die Verwendung eines anderen Begriffs erklärt sich natürlich nicht aus der Notwendigkeit heraus, einer Vielzahl der in der Linguistik existierenden Bezeichnungen für Wortverbindungen eine weitere hinzuzufügen, sondern aus der anhand der Textarbeit gewonnenen Erkenntnis, dass die am Material moderner Sprachen erarbeiteten Begrifflichkeiten - die gängigsten davon sind Phrasem, Phraseologismus, construction - sich nicht ohne Einschränkungen auf das historische Material übertragen lassen. Alleine die zwei bis jetzt für zentral gehaltenen Merkmale eines Phraseologismus bzw. Phrasems, nämlich Polylexikalität und Festigkeit, müssen aus historischer Perspektive revidiert werden: Polylexikalität kollidiert mit den wenig konventionalisierten und verbindlichen Normen der Rechtschreibung, die mal die Zusammen-, mal die Getrenntschreibung ein und desgleichen Belegs erlaubte. Festigkeit ist in historischen Daten mit ausgeprägter Variation als Normal- und Regelfall eher eine seltene Ausnahme. Im Gegensatz zu diesen Termini ist der Begriff „formelhafte Wendung“ weiter gefasst und bezieht sich auf typologisch heterogene Einwortausdrücke, Kombinationen aus mehreren Konstituenten bzw. auf ganze Texte, die holistisch verstanden werden müssen, sich durch ihre starke Funktionalisierung im Kommunikationsprozess auszeichnen, auf Gebrauchskonventionen einer Sprachgemeinschaft beruhen, deren etablierte kulturelle (auch kommunikative) Wissensbestände sie tradieren, und sich auf unterschiedlichen (auch noch nicht abgeschlossenen) Stadien der Konventionalisierung befinden. Im Vergleich zu „construction“ ist der Begriff hingegen enger zu verstehen, da „constructions“ bekanntlich u. a. auch Flexions- und Wortbildungsmorpheme, Komposita sowie abstrakte grammatische Erscheinungen (z. B. analytisches Futur oder Perfekt im Deutschen) einschließen, die alle keine formelhaften Wendungen im hier definierten Sinn sind. Formelhafte Wendungen sind auch nicht unbedingt abstrahierte, auf Kognition basierende Form/Bedeutungspaare, sondern konventionalisierte holistische Einheiten der sprachlichen Oberfläche auf der Mikro- und Makroebene des Textes (cf. die Abschnitte 4.1-4.2).

$\mathrm{Zu} \mathrm{c)} \mathrm{Variation} \mathrm{ist} \mathrm{somit} \mathrm{wie} \mathrm{auch} \mathrm{auf} \mathrm{anderen} \mathrm{sprachlichen} \mathrm{Ebenen} \mathrm{eine} \mathrm{der} \mathrm{treibenden} \mathrm{Kräfte}$ des Sprachwandels im Bereich der Formelhaftigkeit. Die Versuche, sie bei formelhaften Wendungen durch Generalisierungen zu modellieren, liegen in der klassischen Phraseologieforschung einerseits kaum vor, werden aber unter dem Etikett der Verfestigungsprozesse seit langem diskutiert (cf. bereits bei Gvozdarev 1981). Mokienkos Bestreben, Varianten eines bestimmten Phraseologismus aus einer Sprache, aus verwandten Sprachen und Dialekten und schließlich auch aus den genetisch nicht verwandten Sprachen um ein strukturell-semantisches Modell (sic!) zu gruppieren, hat die slawische historische Phraseologieforschung lange geprägt (Mokienko 2002). Die abstrakten Modelle fungieren bei diesem Herangehen als „technische“ Werkzeuge, die den Forscher/die Forscherin zur Rekonstruktion des Prototyps einer Wendung und ihrer Etymologie hinführen sollen. Modellierungsversuche ziehen sich wie ein roter Faden auch durch die gesamte Parömiologie (Kuusi 1974; Röhrich und Mieder 1977), ${ }^{16}$ wo sie teilweise auf die Klassifikation Permjakovs (1979/1984) zurückgehen, laut der die auf der Inhaltsebene ähnlichen Sprichwörter (dt. Schmiede das Eisen, solange es heiß ist; ostfrk. Forme den Lehm, solange er feucht ist; hebr. Bereite den Kürbis zu, solange das Feuer noch nicht erlo-

\footnotetext{
${ }^{16}$ Cf. insbesondere Röhrich und Mieder (1977: 60): „In sich ständig wiederholende Strukturmodelle und Satzmuster sind immer wieder neue Inhalte gegossen und damit sprichwörtlich geworden.“
}

ISSN 1615-3014 
schen ist) als Varianten einer invarianten Situation betrachtet werden, die sie entweder bezeichnen oder modellieren. Dass Sprichwörter aufgrund der Ähnlichkeit auf der Ausdrucksebene gruppiert werden können, wurde von Permjakov ebenfalls früh bemerkt, cf. seinen Begriff „formbildende Gruppe“ (auch logische Modelle) anhand der sprachlichen Floskeln wie z. B. Wenn [...] dann, wo [...] da, wie [...] so, wer [...] der usw. Im deutlichen Unterschied zur Konstruktionsgrammatik erfolgten diese Modellierungen allerdings nicht im Sinne eines Form/Bedeutungspaares, sondern im Entweder-Oder-Modus, also entweder auf der Basis der Inhaltsebene oder auf der Basis der Ausdrucksebene. Beides kombiniert Steyer (2012: 300-310) und bestätigt die Existenz solcher Sprichwortmuster und Strukturformeln für das Gegenwartsdeutsche überzeugend mit strengen korpuslinguistischen Methoden. Sie kommt zum Schluss, dass lexikalisch verwandte Sprichwortmuster nur in wenigen Fällen monosem sind (Der Ton/Unterton/... macht die Musik); nur in solchen Fällen stammen die variablen Konstituenten für lexikalische Slots aus einem bzw. eng verwandten Wortfeldern. Die meisten lexikalischen Varianten bringen trotz invarianter Gesamtbedeutung neue Teilbedeutungen ein (Es ist noch kein Meister/Star/Redner vom Himmel gefallen) oder konstituieren ganz andere Bedeutungen (Der Markt/das Geld macht die Musik). Steyer (2012: 310) plädiert dafür, in solchen Fällen auch neue Muster anzunehmen. Vergleichbare Studien am diachronen Material liegen zurzeit nicht vor.

Der Vorteil der Untersuchungen zu Verfestigungsprozessen besteht darin, dass seit Beginn der Diskussion unter dem Stichwort „derivationelle Basis“ die Rolle des Kontextes berücksichtigt wurde (Sialm, Burger und Linke 1982: 323-330): Als solche konnte eine freie Wendung, ein anderer Phraseologismus, ein Text, eine bestimmte Kommunikationssituation (z. B. der Gebrauch in Fachsprachen) bzw. die Verwendung in einer anderen Sprache fungieren. Der Nachteil der älteren Forschung ist die wenig empirisch ausgerichtete textbasierte Vorgehensweise und folglich die Unfähigkeit, die Dynamik in kleineren Schritten zu verfolgen. Der Grund dafür besteht allerdings nicht in der prinzipiellen Ignoranz der genauen Veränderungsschritte, sondern 1) in der Unmöglichkeit ihrer Untersuchung wegen des nicht (ausreichend) vorliegenden Datenmaterials und 2) im grundsätzlichen Problem jeder sprachhistorischen Forschung, nämlich der großen Überlieferungslücken selbst in vorhandenen Textdaten, die das Nachzeichnen ununterbrochener Verfestigungswege erheblich erschweren.

Wenn in der klassischen diachron orientierten Phraseologieforschung Versuche unternommen wurden, abstrakte Modelle zu formulieren, dann richteten sie sich wenig auf die Ableitung einer Konstruktion, die einer Gruppe der Varianten bei einer konkreten Wendung zugrunde liegt, sondern auf die Erarbeitung der generellen Verfestigungstypen. Eine Aufzählung in Burger und Linke (22008: 746-753) schließt solche Prozesse mit ein wie Reduktion von lexikalischen Varianten, Verfestigung der morphosyntaktischen Struktur, z. B. durch die Fixierung der Artikel oder Numerusformen auf jeweils eine Variante, Festlegung auf die Diminutivform, Festlegung auf die affirmative/negative Variante, Fixierung der Wortfolge, Substitution im Konstituentenbestand usw. Schowe (1994) zeigt in einer detaillierten Studie zu Idiomen aus dem Rechtsbereich für die semantische Verfestigung die Prozesse der Idiomatisierung durch Bedeutungsabschwächung, Idiomatisierung bei gleicher Bedeutung, Idiomatisierung durch Abstrahierung usw. Das Augenmerk liegt zwar eindeutig immer auf einem Bereich der phraseologischen Oberfläche, aber der ebenenübergreifende Charakter des Wandels ist auch diesen Ausführungen nicht fremd. So stellen Burger und Linke ( ${ }^{2} 2008$ : $747 \mathrm{f}$.) beim Idiom ein Auge auf jemanden

ISSN 1615-3014 
werfen neben der heute üblichen Fixierung des unbestimmten Artikels und der Singularform auch die Bedeutungsverengung fest: Von den ursprünglichen Bedeutungen a), etwas/jemanden (genau) ansehen, betrachten' und b) ,Interesse für etwas/jemanden zeigen/Gefallen finden an jemandem/etwas' ist nur noch die erste erhalten geblieben. Die morphosyntaktische Verkürzung der frühneuhochdeutschen Paarformel über Hals und Kopf läuft vermutlich parallel zum allmählichen Verblassen der zugrundliegenden höchst konkreten Vorstellung (also der bildlichen Grundlage) des mit dem Kopf Vornüberstürzens und sich dabei Überschlagens (ebd.: 748). Hüpper, Topalovic und Elspaß (2002) gelingt es, für Paarformeln im Rechtskontext nachzuweisen, dass sie dort lexikalisch bereits sehr früh fest sind, weil ein vereinheitlichendes Recht wie der großräumig über Jahrhunderte wirkende Sachsenspiegel sprachliche Normen vorgibt; morphosyntaktische Variabilität (die Umkehrung der Reihenfolge der Konstituenten) bleibt hingegen lange in Abhängigkeit des syntaktischen Kontextes und der Stilistik erhalten. Daraus leiten die Autoren eine Abfolge der Phraseologisierungsschritte im Sinne eines „Phraseologisierungspfades“ ab: 1) Festlegung des Lexembestandes, 2) Entwicklung einer Reihenfolgepräferenz, die gerade nicht durch das Behaghel'sche Gesetz der wachsenden Glieder oder Müllers Salienzkriterien bestimmt wird, und 3) Festigung der Morphosyntax (ebd.: 96). Die Veränderung des außersprachlichen Kontextes (z. B. die Abschaffung der gesetzlich geregelten Bestrafungsmethoden wie im Fall von jemanden auf die Folter spannen) setzt eine (freie) Wortverbindung aus dem rechtlichen Bereich als Bildspender für eine neue - idiomatische - Bedeutung (in diesem Fall, jemanden neugierig machen') in der Allgemeinsprache frei (Schowe 1994: 210). In die Nähe der Konstruktionsgrammatik kommt die von Burger (2012: 15) geäußerte Beobachtung: „Von Variation in der Phraseologie zu sprechen, hat nur einen Sinn, wenn hinter den Varianten eine in gewissem Maße stabile Einheit erkennbar ist. Diese Einheit ist grundsätzlich semantisch zu bestimmen“.

Hier wird die Existenz eines Modells, eines Schemas oder eben einer Konstruktion angenommen, die im Sinne einer Abstraktion/Generalisierung als übergeordnetes Dach fungiert und die nach einem ähnlichen Muster entstehenden Varianten zusammenführt. Aus der Perspektive der Konstruktionsgrammatik wäre zu ergänzen, dass diese Generalisierung nicht nur semantisch, sondern auch kognitiv und sozial zu bestimmen wäre, bei allen Schwierigkeiten, die diese Kategorien für diachrone Studien mit sich bringen.

Als Zwischenfazit zu Abschnitt 3 lässt sich festhalten, dass die historische Phraseologieforschung unter dem Stichwort „Verfestigungsprozesse“ bereits seit langem Fragen diskutiert hat, die die diachrone Konstruktionsgrammatik sich gegenwärtig auch stellt. Bis jetzt haben diese Untersuchungen allerdings nicht zur eigenen linguistisch fundierten Theoriebildung geführt. Was die Erforschung der historischen formelhaften Wendungen für diachron orientierte Konstruktionsgrammatiken nichtdestotrotz wertvoll macht, ist die Tatsache, dass die erstere durch die Untersuchung der Verfestigungsprozesse ganz andere Faktoren des Sprachwandels ins Spiel bringt, weil sie im Bereich der Formelhaftigkeit anders als in der Phonetik, Morphologie, Lexik oder Syntax gelagert sind. Das Belegen u. a. auch folgende Erkenntnisse des HiFoS-Projekts:

Erstens geht die Verfestigung nicht immer automatisch mit der Reduktion der Varianten einher, sondern korreliert paradoxerweise mit ihrer Zunahme, beispielsweise bei Kollokationen und Modellbildungen (Burger 2012: 15). 
Zweitens verläuft der Wandel nicht immer nach dem Prinzip der Ökonomie in Richtung Vereinfachung der Struktur. Dies wird besonders bei Funktionsverbgefügen deutlich, die im älteren Deutsch semantisch wenig spezifische Verben als Konstituenten in ihrer Struktur hatten, in der modernen Sprache hingegen lexikalisch hochspezifiziert sind, cf.: die frühneuhochdeutschen Beispiele einen Angriff thun, Einfälle thun, Widerstand thun, einen Vorschlag thun, eine Forderung thun, eine Reise thun, eine Fahrt thun, einen Fußfall thun (Burger 2012: 12f.), die heute mit anderen verbalen Konstituenten konventionalisiert sind.

Drittens verlaufen die Verfestigungsprozesse in den seltensten Fällen linear von wenig Idiomatizität zu mehr Idiomatizität bzw. von wenig Festigkeit zu mehr Festigkeit. Genauso wie bei Grammatikalisierungs- und Lexikalisierungsprozessen kann die Entstehung der Formelhaftigkeit auf bestimmten Stufen aufhören und unvollendet bleiben; im Gegensatz zu diesen braucht sie aber nicht unidirektional und irreversibel zu sein. So kann z. B. die opak gewordene bildliche Grundlage eines Idioms re-motiviert werden. Burger (2012: 12) zeigt dies am Beispiel der Konstituente Kegel in der Paarformel mit Kind und Kegel: Die metonymisch gemeinte Bedeutung ,außereheliches Kind' trug im älteren Deutsch zur Gesamtbedeutung ,mit ehelichen und außerehelichen Kindern'> , mit allem, was man hat ' bei und ist heute nicht mehr lebendig; die Konstituente wird allerdings als ,Figur des Kegelspiels‘ umgedeutet, aufgrund dessen auch die gesamte Wendung wieder metonymisch als ,mit allem Wichtigen und Unwichtigen ' entziffert werden kann.

Viertens ist die Reduktion der Varianten im Bereich der Formelhaftigkeit nicht von der Kodifikation abhängig. Die Rolle dieses Faktors wurde im Bereich der Aussprache und Rechtschreibung in Kohrt ( $\left.{ }^{2} 1998\right)$, im Bereich der Grammatik in Werner $\left({ }^{2} 1998\right)$ und Mattheier $\left({ }^{2} 2000\right)$ betont. Da aber phraseologische Wörterbücher zu grundsätzlich anderen Zwecken (z. B. nicht zum Zweck der sprachlichen Normierung) als Rechtschreibwörterbücher oder Grammatiken erarbeitet wurden und immer noch werden, muss das Einwirken der Kodifizierung hinterfragt werden. In Filatkina (im Druck) wurde die Divergenz zwischen zwei Überlieferungssträngen den älteren Primärtexten und kodifikatorischen Quellen (Wörterbüchern, sprichwörtlichen Sammlungen, historischen Grammatiken) - nachgewiesen. Historische wie moderne Wörterbücher tradieren einen Bestand an formelhaften Wendungen und ihrer Variation, der sich teilweise stark davon unterscheidet, was in Primärtexten überliefert ist. Somit vermitteln die Wörterbücher ein anderes Bild über die Variation im Bereich der formelhaften Wendungen, was nicht besagt, dass dieses Bild ein falsches ist, aber es unterscheidet sich eben deutlich von der Varianz, die sich in Texten entfaltet. Deshalb scheint die Kodifizierung im Gegensatz zu anderen sprachlichen Ebenen bei Verfestigungsprozessen der formelhaften Wendungen eine geringere Rolle zu spielen.

Fünftens zeigen formelhafte Wendungen, dass bei ihrer Dynamik und dem Wandel die Veränderungen des außersprachlichen Kontextes eine wichtigere Rolle als z. B. beim grammatischen Wandel spielen und unbedingt berücksichtigt werden müssen. Die Idiomatisierung von Aller guten Dinge sind drei, etwas auf die lange Bank schieben oder Wer A sagt, muss auch B sagen wird nur dank der Statusveränderung dieser Wendungen im rechtlichen Kontext des Mittelalters ermöglicht, die sie für den Übergang in die allgemeine Sprachverwendung, wo die Idiomatisierung ja eigentlich auch erst stattfindet, öffnen (Schowe 1994: 217-224). Die Verankerung bzw. die Herkunft der Wendungen aus großräumig verbreiteten Texten wie etwa dem Sachsenspiegel oder der Bibel begünstigen ihre Verbreitung, aber auch ihre Verfestigung. Hüpper, Topalovic 
und Elspaß (2002) weisen dies für Paarformeln nach, Piirainen (2012) für figurative Wendungen, die heutzutage zu weitverbreiteten Idiomen quer durch die Grenzen der genetisch verwandten Sprachen gehören. Eines dieser Idiome ist das oben bereits erwähnte Beispiel Perlen vor die Säue werfen (Dobrovol'skij und Piirainen 2009: 35f.; Piirainen 2012: 231-235), bei dem die biblische Herkunft die große Verbreitung bereits im frühen Mittelalter erklärt und den Erhalt der zumindest im Deutschen und Niederländischen weniger frequenten Konstituente Säue (statt Schweine) verdeutlicht - Säue ist das Wort, das Luther in seiner Bibelübersetzung verwendet. Auch Besch (1998) führt die Verfestigung der Konstituente Scheffel in sein Licht (nicht) unter den Scheffel stellen auf die Wahl dieses Lexems durch Luther und sein (wohl auch dadurch) gestiegenes großräumiges Durchsetzen gegen regionale Varianten Müdde/Mutt/Mütt, Sumber/Simmeryn/Simmer, Sester/Sechter, Metze, Viertel u. a. zurück. Einen Fall von langfristiger Verweigerung belegt Besch (1999) hingegen mit dem Idiom wider/gegen den Stachel löcken: das ursprüngliche kleinräumige Lexem lecken wird im Zuge der Verfestigung trotz Luthers Wortwahl nicht angenommen. Soweit ich sehe, fangen solche außersprachlichen Faktoren erst an, in konstruktionsgrammatischen Diskussionen eine Rolle zu spielen; ihre Berücksichtigung wird programmatisch gefordert, gleichwohl wird auch darauf hingewiesen, dass die nötigen Analysekategorien noch fehlen. ${ }^{17}$

\section{$4 \quad$ Fallstudien am Material des Althochdeutschen (Ahd.)}

Die analysierten Vorteile, aber auch die Grenzen konstruktionsgrammatischer Ansätze bei diachronen Untersuchungen von formelhaften Wendungen möchte ich im Folgenden an einigen Beispielen weiterdiskutieren. Im Mittelpunkt stehen vor allem solche formelhaften Wendungen, die im modernen Deutsch entweder als semantisch und syntaktisch fest gelten oder nicht mehr existieren, aus diachroner Perspektive aber den Einfluss der Faktoren aufzuzeigen erlauben, die bis jetzt wenig seitens der Konstruktionsgrammatiken berücksichtigt wurden.

\subsection{Verbsagen (und) Verbsagen}

Mein erstes Beispiel ${ }^{18}$ ist eine nach dem Muster VERBsagen (und) VERBSagen gebildete formelhafte Wendung:

(1) et dicit ei petrus. | domine tu mihi lauas pedes. | respondit ihesus | et dicit ei. quod ego facio | tu nescis modo. Inti quad imo petrus | trohtin uuesgistu mir nu fuozzi. | tho antlingita ther heilant $\mid$ Inti quad imo thaz ih tuon $\mid$ thaz niuueistu nu. (Tatian 270, 4 Io; ID 29395)

[Und es sagte ihm Petrus: „Herr, wäschst du mir nun die Füße?“ Da antwortete der Heiland und sprach zu ihm: „Dass/Ob ich es tue, das weißt du nicht.“"]

\footnotetext{
17 Cf. exemplarisch für die Synchronie Fried 2009b und Ziem 2015, für die Diachronie - Filatkina 2015; 2018; Fried 2009a, 2013; Östman und Trousdale 2013 und Weber 2015.

${ }_{18}$ Die Notation der Beispiele erfolgt nach den Prinzipien der HiFoS-Nachwuchsforschergruppe. Zum Zweck der Vergleichbarkeit ist der althochdeutschen Stelle ein Ausschnitt aus der lateinischen Vorlage beigegeben. Die Übersetzung ins Neuhochdeutsche in eckigen Klammern erleichtert das Verständnis, erhebt keinen literarischen Anspruch, sondern dient der möglichst textgenauen Wiedergabe der althochdeutschen Stelle. Der eigentliche Beleg ist kursiv hervorgehoben. In runden Klammern finden sich die genauen Angaben der Belegstelle im Text und die ID-Nummer des Belegs in der HiFoS-Datenbank.
} 
Mit 40 Belegstellen ist das Muster für ein diachrones Korpus gut verbreitet, nach dem heutigen Kenntnisstand ist es nur in ahd. Texten überliefert und gerät später außer Gebrauch. Die absolute Mehrheit der ahd. Belege (insgesamt 32) entfallen auf den Tatian - eine lateinisch-althochdeutsche Bilingue aus dem 2. Viertel des 9. Jahrhunderts. Fünf weitere Stellen finden sich im Isidor, zwei in der Althochdeutschen Benediktinerregel. ${ }^{19}$ Damit sind Texte genannt, die keine autochthonen deutschen Textdenkmäler sind, sondern zu der Gruppe der religiösen Übersetzungsliteratur gehören. Aus der autochthonen Literatur liegt uns kein einziger Beleg vor. Wie auch schon Beispiel (1) annehmen lässt, handelt es sich in diesem Fall um eine Lehnübersetzung aus dem Lateinischen respondens ... ait/dixit bzw. respondit et dixit. Diese Wendung ist im Ahd. hinsichtlich ihrer Semantik/Pragmatik fest und folgt dem lateinischen Pendant: Als ein Textgliederungssignal führt sie ein Zitat bzw. eine direkte Rede ein, cf. Beispiel (1). Fest ist ferner das zugrunde liegende Muster; dessen lexikalische Slots sind in dem Sinn variabel, als sie durch unterschiedliche Verben des Sagens gefüllt werden können, die ihrerseits in Bezug auf die grammatischen Kategorien Tempus, Numerus, Person, Finitum/Infinitum variieren. Die Beispiele in (2) geben einen Überblick über das Variationspotenzial und sind absteigend nach dem Grad der Verbreitung der Belege geordnet:

(2a) antuurtita (inti) quad [antwortete (und) sagte]

antuurtenti (inti) quad [antwortend (und) sagte]

antuurtita (...) quad (...) quedenti [antwortete (...) sagte (...) sprechend]

(2b) antlingita (...) (inti) quad [antwortete (...) (und) sagte]

antlinginti (...) quad [antwortend (...) sagte]

(2c) (fora) chundida (inti) quhad [verkündete (und) sprach]

(2d) bat (...) (sus) quedente [bat (...) (so) sprechend]

(2e) betota (...) quedenti [betete $(. .$.$) sagend]$

(2f) reof quedenti [rief sagend]

(2g) $\operatorname{sprah}(. .$.$) (sus) quedenti$ [sprach $(\ldots)$ so sagend] respondens $[\ldots]$ ait/dixit

respondens [...] ait/dixit

respondens $[\ldots]$ dixit $[. .$.$] dicens$

respondit [...] et dicit

respondens [...] dixit

praenuntiauit [...] dicens

rogabat [...] dicens

adorauit $[\ldots]$ dicens

clamauit dicens

locutus est [...] dicens

\footnotetext{
${ }^{19}$ Cf. auch AWB (1, 573 und 552). Einige wenige Belege mit antuurten finden sich auch in Notkers Bearbeitung von Boethius De consolatione philosophiae, wobei die verbale Konstituenten quedan hier durch die direkte Rede von der ersten Konstituente antuurten abgetrennt ist und das pronominale Subjekt zweimal vorkommt, cf. etwa die Stelle to antuuurta er imo ,Ia got ' chad er (Nb 117, $1[127,20])$.
} 
(2h) predicando (...) quhad

[voraussagend (...) sprach]

(2i) sprechente (...) inti quadun

[sprechend (...) und sagten]

(2j) heftita (...) quhad

[bezog sich (...) sagte]

(2k) keaugit (...) qhuedenti

[zeigt (...) sagend] predicat $[\ldots]$ dicens

loquentes $[\ldots]$ et dicebant

ohne Vorlage

ostendit [...] dicens

Zwischen ahd. antwurten (2a) und ahd. antlingen/antlingōn (2b) lässt sich kein semantischer Unterschied feststellen; innerhalb dieser Konstruktionen fällt allerdings auf, dass antwurten meistens in der 3. P. Sg. Prät. Ind. für lat. Part. Präs. respondens steht; antlingon entspricht hingegen der 3.P. Sg. Perf.-Form respondit. Folgen im Lat. beide Verbformen öfter parataktisch unmittelbar aufeinander ab, sind sie im Ahd. durch andere Konstituenten, z. B. durch die Konjunktion inti, den Dativ der Person imo, durch präpositionale Verbindung zi in, getrennt. Die Form des finiten Verbs ist im Ahd. auf Präteritum (außer in 2k), des infiniten - auf Partizip Präsens beschränkt; im Lat. sind auch andere Tempusformen möglich (cf. Imperfekt und Perfekt).

Im Sinne der Konstruktionsgrammatik können die Varianten (2a) bis (2k) als Mikrokonstruktionen der abstrakten Makrokonstruktion Verbsagen (und) Verbsagen in der Bedeutung, Einleitung eines Zitats, einer direkten Rede“ verstanden werden. Die Variation in der lexikalischen Besetzung und der morphosyntaktischen Struktur zieht allerdings auch synchron im Ahd. keine Variation im Bereich der Semantik nach sich, was zu erwarten wäre, wenn man die Beispiele als Form-/Bedeutungspaare beschreiben wollte. Die konstanten aus dem Lateinischen entlehnten Bedeutung und Funktion sind hier viel mehr an eine (sowohl bereits im Lateinischen als auch im Ahd.) lexikalisch und morphosyntaktisch variable Form gebunden. Der Begriff Form-/Bedeutungspaar kommt in meinen Augen erst auf der Makroebene der abstrakten Generalisierung zum Tragen.

\subsection{Präp $+n \bar{o} t$}

Das nächste Beispiel weist hingegen eine feste Form bei ausgeprägter semantischer Variation und Vagheit auf. Es handelt sich um eine im Ahd. adverbial verwendete geläufige Verbindung Präp $+n \bar{o} t$, in der nur die erste Konstituente (die Präposition) variabel ist und die zweite in Form des Substantivs nōt völlig fixiert ist, z. B. b̄̄ nōti/bī nōtim, zi nōti, in nōti, fona nōti, thuruh $n \bar{t}$, āna nōt. Mit über 100 Belegstellen ist die Konstruktion im Ahd. mit ihren Varianten weit verbreitet, wobei sich bei ihrer Verfestigung der Einfluss des Lateinischen nicht feststellen lässt. Das hohe Variationspotential erklärt sich hier in meinen Augen durch die semantische Vagheit der beiden Konstituenten, die auch durch die Konventionalisierung zu einer Wendung nicht eingeschränkt zu sein scheint. Für das Lexem nōt in freier Verwendung gibt Schützeichel ( ${ }^{6}$ 2006: 256) folgende Bedeutungen an: ,Not, Notlage; Zwang, Gewalt, Bedrängnis; Bedarf, Bedürfnis; Bedürftigkeit, Hilflosigkeit; Entbehrung; Gefahr, Verfolgung; Kampf; Gericht; Pein, Qual; Angst, Trübsal; Kürze; Heftigkeit; Grund, Notwendigkeit‘. Davon, dass die Präpositionen im Ahd. ebenfalls semantisch kaum festgelegt sind und über einen niedrigen Grad an 
Grammatikalisierung verfügen, zeugen am besten die entsprechenden Artikel im AWB. Natürlich trägt der unmittelbare Kontext in einigen Fällen zur Disambiguierung der Wendung bei, für jede formale Variante kann allerdings nur eine mehr oder weniger prototypische Bedeutung formuliert werden; Beispiele, die semantische Ähnlichkeiten zwischen den aufgezählten Varianten belegen, lassen sich aber auch reichlich finden. Die prototypischen Kontexte sind in (3) bis (8) zusammengestellt:

(3a) bi nōti $i_{1}$,kommentierend, charakterisierend: notwendigerweise, naturgemäß, wie es sich gehört, den Umständen entsprechend‘

Oba ther man uuesti ther heime ist in ther festi $\mid$ al thaz ungizami · uuio ther thiob quami - | Er uuacheti bi noti . thanne in theru ziti | dribi then thiob thanana uz $\cdot$ ni liazi irgraban sinaz hus. (Otfrid, Evangelienbuch, 122v, IV 7, 57; ID 11144)

[Wenn der Mann, der daheim im Haus ist, von all dem Missgeschick wüsste, wie der Dieb käme, würde er notwendigerweise dann zu dieser Zeit wachen [und] den Dieb hinaus treiben. Er würde nicht sein Haus aufbrechen lassen.]

(3b) bi nōti , kommentierend, charakterisierend: notwendigerweise, aus Not, gezwungenermaßen, unter Zwang‘

Purpurin giuuati . druag er tho bi noti | thurnina corona . gidan uuas thaz in hona. (Otfrid, Evangelienbuch, 139v, IV 23, 7; ID 10799)

[Ein purpurnes Gewand trug er da gezwungenermaßen [und eine] Dornenkrone, dies wurde ihm angetan zum Spott.]

(3c) bi nōti , kommentierend, charakterisierend: notwendigerweise, unbedingt, nachdrücklich, eindringlich

Vuoltun tho thie liuti $\cdot$ fahan nan bi noti $\mid$ giuuisso thaz ni hilu ih thih $\cdot$ duan zi kuninge ubar sih. (Otfrid, Evangelienbuch, 81v, III 8, 1; ID 10620)

[Die Leute wollten ihn da unbedingt empfangen, gewiss verhehle ich dir das nicht, [um] ihn zum König über sich zu machen.]

(4a) fona $n \bar{t} t i_{1}$,kommentierend, charakterisierend: notwendigerweise, notgedrungen, aus Notwendigkeit'

Vuanda doh fiê beîde Góte mif|felîchen . die fóne nôte unde âna nôt mífletuont . dâr ift doh | ána fílo gefceîdenef. (Notker, Psalmenauslegung, 256, 1; ID 8969)

[Denn obwohl sie beide Gott missfallen mögen, die aus Notwendigkeit und [die] ohne Notwendigkeit sündigen, ist doch dabei viel Unterschiedliches.]

(4b) fona $n \bar{t} t i_{2}$,kommentierend, charakterisierend: notwendigerweise, naturgemäß, wie es sich gehört' 20

$\{Q\}$ UOD SI HĘC NON SUNT POSSIBILIA . S . | ut omnia ex necessitate fiant . Ube | dés nîeht uuésen nemág. táz álliu ding fóne nôte geskéhen. (Notker, De interpretatione; 181, 1; ID 4010)

\footnotetext{
${ }^{20}$ AWB $(3,1109)$ verweist explizit auf den formelhaften Charakter der Wendung, gibt allerdings nur die Bedeutung , mit/aus Notwendigkeit, notwendigerweise' an.
} 
(5) āna nōt ,kommentierend, charakterisierend: ohne Mühe, mühelos, willig, gern“

Omnia quęcumq[ue] uoluit $\mathrm{d}[\mathrm{omi}] \mathrm{n}[\mathrm{u}] \mathrm{f}$ fecit in cẹlo [et] in terra . in mari [et] | in omnibuf abyflif. Vnfer truhten téta alliu diû er uuolta $\mid$ in himele unde in erdo . unde in allên uuázeren . âne nôt. (Notker, Psalmenauslegung, 502, 7; ID 9154)

[Alle, die er auch immer wollte, machte der Herr im Himmel und auf der Erde, im Meer und in allen Tiefen. Unser Herr machte all, die er wollte, im Himmel und auf Erden, und in allen Gewässern gern.]

(6) zi nōti ,kommentierend, intensivierend: eifrig, mit Eifer, ganz und gar, sehr ${ }^{6}$

Sie uuegitun iro houbit . ioh sprachun ubilo tharmit $\mid$ sprachun tho zi noti . thaz iro heizmuati (Otfrid, Evangelienbuch, 146r, IV 30, 8; ID 11044)

[Sie [= alle anwesenden Leute und die Priester] schüttelten ihre Köpfe und schmähten [ihn] auch damit, [sie] sprachen da eifrig ihren Hass [aus].]

(7a) in nōti , bestätigend, bekräftigend: in der Tat, tatsächlich“

Druhtin selbo in uuara . uuaz zellu ih thir es mera $\mid$ ioh thie mit imo in note . uuarun uuallonte $\mid$ Sint sie untar mennisgon . after gote furiston $\mid$ in himilriche ouh thaz ist uuar (Otfrid, Evangelienbuch, 125r, IV 9, 26; ID 11147)

[Der Herr selbst fürwahr (was erzähle ich dir davon mehr?) und die mit ihm in der Tat umhergezogen waren, sie sind unter den Menschen nach Gott auch Fürsten im Himmelreich, das ist wahr.]

(7b) in $n \bar{t} t i_{2}$, charakterisierend, kommentierend, intensivierend: mit Eifer, eifrig ${ }^{6}$

Uuanta ih zellu in noti $\cdot$ iro armilichun dati $\mid$ thio missidat so sagen ih · bi thiu inkunnun sie mih (Otfrid, Evangelienbuch, 91v, III 15, 31; ID 10735)

[Denn ich berichte eifrig von ihren erbärmlichen Taten, die Missetat, wie ich sage. Daher hassen sie mich.]

(7c) in nōti $i_{3}$,kommentierend, charakterisierend: in der Not, in einer schweren Lage

In tribulatione inuoca|ui $\mathrm{d}[\mathrm{omi}] \mathrm{n}[\mathrm{u}] \mathrm{m}$. [...] In nôte unde in éngi há|reta ih ín ána . [...] (Notker, Psalmenauslegung, 433, 13; ID 7574)

[Im Kummer rief ich den Herrn an. [...] In Not und Bedrängnis rief ich ihn an.]

(8) thuruh $n \bar{o} t$, charakterisierend, kommentierend: eifrig, willig, eindringlich, nachdrücklich'

$\mathrm{Ni}$ uuanu iz uuola intfiangin . ioh nahor ouh gigiangin | fragotun sie avur thuruh not $\cdot$ so man in heime gibot | Oba thu Helias avur bist · ther uns kunftiger ist $\mid$ thaz gizeli thu uns nu sar · thaz uuir iz avur sagen thar (Otfrid, Evangelienbuch, 38v, I 27, 22; ID 23614)

[Ich glaube nicht, dass sie es gut aufnahmen und sich auch näher darauf einließen. Sie fragten wiederum willig, wie man es ihnen zu Hause geboten hatte: „Ob du aber Elias bist, der zu uns kommen wird, das erzähle uns nun gleich, damit wir es dort wiederum sagen können."] 
Die in dieser Gruppe geläufigste Wendung (3) kommt in drei prototypischen Gebrauchskontexten vor und tradiert in (3a) und (3b) die typischen Bedeutungen ,Not als missliche, schwierige Lage“ und ,Not und Zwang' des Lexems $n \bar{o} t$ in der freien Verwendung; die Präposition $b \bar{\imath}$ wird modal im Sinne von , gemäß, mit, nach, zu'verwendet (AWB I, 969). Die kommentierende bzw. charakterisierende Funktion der Belege ist konstant. Semantisch-pragmatische Unterschiede zu fona nōti $(4 \mathrm{a}, \mathrm{b})$ lassen sich kaum feststellen. Im Beleg-Korpus der HiFoS-Gruppe ist fona nōti allerdings auf die Texte Notker des Deutschen beschränkt, bī nōti ist hingegen für Otfrids Evangelienbuch typisch. Bei āna nōt (5) können zwar auch mindestens zwei Bedeutungen unterschieden werden: Die Wendung weist in (4a) als Gegenpol zu fona nōti die Bedeutung ,ohne Not' auf; allerdings ist die Zahl solcher Belege zumindest im HiFoS-Belegkorpus deutlich den Belegen mit der Paraphrase (5) ,ohne Mühe, mühelos, willig, gern' unterlegen.

Im Unterschied zu den Beispielen (3) bis (5) liegt der aktuellen Bedeutung der Wendungen in (6) bis (8) wohl eine andere Bedeutung des Lexems $n \bar{o} t$ zugrunde. Gemeint ist eher die gegenwärtig verschwundene Bedeutung von nōt im Sinne von ,Heftigkeit', die in meinen Augen auch teilweise die intensivierende Funktion der gesamten Wendung mit beeinflusst. Während sie bei den Belegen in (3c) nur implizit mitschwingt, ist sie in den Beispielen (6) bis (8) zentral. Die Analyse des HiFoS-Belegkorpus' liefert allerdings keine eindeutige Antwort auf die Frage, wodurch sich die Belege innerhalb dieser Gruppe semantisch/pragmatisch voneinander unterscheiden. Die Bedeutung ,aus Not, in der Not, in einer schweren Lage‘ lässt sich bei in nōti (7c) mit einer ausreichenden Zahl der Belege dokumentieren; durch die Kombination mit in engi, in Bedrängnis' wird sie besonders deutlich. thuruh nōt ist in der HiFoS-Datenbank auch in der Bedeutung ,notwendigerweise, aus einem äußeren oder inneren Zwang heraus‘ belegt, die auch das AWB $(2,780)$ bestätigt. Hingegen findet sich die in (8) angegebene Paraphrase nicht im AWB, das Wörterbuch vermerkt aber gerade in Bezug auf die Verwendung der formelhaften Wendung in Otfrids Evangelienbuch, dass diese „bei Otfrid oft kaum übersetzbar“ sei.

Für die synchrone Beschreibung der Variation bei der Konstruktion Präp + nōt im Ahd. lässt sich der begriffliche Apparat der Konstruktionsgrammatik also prinzipiell auch ansetzen. Das Netzwerk an Mikrokonstruktionen, die der Makrokonstruktion Präp + nōt zugeordnet werden können, unterscheidet sich von dem in Abschnitt 4.1 beschriebenen Netzwerk. Es ist vielfältiger und erlaubt auch die Annahme der Zwischenebene der Mesokonstruktionen (bī nōti/bī nōtim, zi nōti, in nōti, fone nōti, thuruh nōt, āna nōt), deren formales Muster fest ist und sich auf den ersten Blick nur durch die Verwendung unterschiedlicher Präpositionen unterscheidet. Aufgrund der Unterschiede in der Semantik und pragmatischen Funktion können zwei größere Gruppen von diesen Mesokonstruktionen unterschieden werden: (3) bis (5) vs. (6) bis (8), wobei die Unterschiede hier weniger durch den Kontext und gar nicht durch Sprachkontakt erklärt werden können, sondern eher durch die Aktualisierung unterschiedlicher Bedeutungen der zweiten substantivischen Konstituente. Sie sind sozusagen wendungsinterner Art. Damit ist ein potentieller Auslöser der Konstruktionalisierung genannt, der in den rezenten Diskussionen kaum eine Rolle gespielt hat. Die Analyse der Beispiele (3) bis (8) zeigt zusätzlich wiederum die Problematik der Definition der Konstruktionen als Form-/Bedeutungspaare, will man diesen auf die mittlere Ebene der Mesokonstruktionen beziehen. Innerhalb der Gruppen sind die Wendungen polysem, was oben in (3), (4) und (7) explizit gezeigt wurde, aber auch für andere Gruppen gilt. Die Form der Mesokonstruktionen (3), (4) und (7) bleibt aber unverändert: bi nōti

ISSN 1615-3014 
in (3a, b, c), fona nōti in (4a, b, c) und in nōti in (7a, b). Es handelt sich hier also um eine semantische Polysemie, der aber keine morphologische Variation folgt. Auch wenn Goldberg (1995: 31-39) die Polysemie als eine der wichtigsten Eigenschaften der ditransitiven Konstruktionen behandelt und die zentrale Bedeutung (central sense) von verwandten Bedeutungen (related senses) unterscheidet, sieht sie die Polysemie in den unterschiedlichen Möglichkeiten der lexikalischen Besetzung des verbalen Slots begründet, mit anderen Worten: semantische Veränderungen gehen mit formalen einher. So evozieren die Verben des Gebens (give, pass, hand, serve) z. B. die zentrale Bedeutung der ditransitiven Konstruktionen, die Verben der Erlaubnis (permit, allow) - eine der verwandten Bedeutungen. Die morphologische Variation bzw. die Veränderung der Form kann bei den Mesokonstruktionen (3), (4) und (7) wie bemerkt nicht festgestellt werden, die Bedeutung variiert hingegen. Welche Bedeutung innerhalb der Gruppen die zentrale und welche die verwandte ist, lässt sich ebenfalls nicht sagen. Der konstruktionsgrammatische Begriff würde an Erklärungspotential gewinnen, wenn er (auf der Ebene der Mesokonstruktionen) nicht im strengen Sinn der Zuordnung, eine Form - eine Bedeutung` zu verstehen wäre.

\subsection{Diachrone Formulierungstraditionen und „Textkonstruktionen“}

Formelhaftigkeit ist nicht nur ein Merkmal des konkreten sprachlichen Ausdrucks; sie lässt sich auch auf der Makroebene der Textorganisation beobachten. Spätestens seit sich die Textlinguistik unter dem Einfluss der kognitionspsychologischen Erkenntnisse über die Rolle der Schemata, Rahmen bzw. Skripte bei der Wissensaneignung, -organisation, -speicherung, -produktion und -verwendung das Verständnis von Textkonstitution als kognitiven Vorgang und von Texten als Ergebnisse mentaler Vorgänge zu eigen gemacht hat, gehört das Wissen über so genannte „globale Muster“, „komplexe Muster“ oder „Textmuster“ (Heinemann und Heinemann 2002: 129-165) zu den festen Größen im Forschungsprogramm, insbesondere im Rahmen der Kontextualisierungstheorie (Fillmore 1976, Gumperz 1982) und der Formulierungsund Textproduktionsforschung (Antos 1982, 1989). Bereits van Dijk (1980) spricht von „konventionalisierten Darstellungsstrukturen, die per Konvention eingehalten werden“. In Anlehnung an soziologische Diskussionen (stellvertretend Luckmann 1986; Berger und Luckmann 1966/2010) geht Feilke davon aus, dass jedes kommunikative Handeln, also auch das Sprechen, durch eine Typik des Verhaltens erkennbar ist. Sprachhandeln muss u. a. als Prozess einer Institutionalisierung von kulturellen und sprachlichen Schemata über die Schritte der Typisierung, Schematisierung und Habitualisierung, als ,Überführung kontingenten Ausdrucksverhaltens in einen sozial strukturierten semiotischen Habitus“ (Feilke 1994: 209f.) verstanden werden. Eine besondere Rolle wird dabei dem Common sense-Wissen beigemessen. Dieses ist „ein auf die ökologischen Bedingungen menschlichen Handelns bezogenes und durch diese Bedingungen pragmatisch konstituiertes und stabilisiertes intuitives Wissen" (Feilke 1994: 363), ein „kognitives und moralisches Sediment unserer alltäglichen Erfahrungsprozesse“ (Feilke 1994: 362). ${ }^{21}$ Das habitualisierte Weltwissen bezieht sich nicht nur auf das gemeinsam erzeugte Wissen über bestimmte Handlungsabläufe (z. B. Szenarien und Abläufe eines Festakts), sondern hat auch seine linguistische Seite. Warnke (1999: 219) beobachtet in verschiedenen Texten und

${ }^{21}$ Cf. ähnlich auch Feilke (2012) in Bezug auf schriftliche Textroutinen.

ISSN 1615-3014 
Diskursen „standardisierte Formen verbalen Handelns“, die „,der formalen und inhaltlichen Organisation von sprachlichen Handlungen im Hinblick auf jeweilige Funktionen“ dienen. Die Muster oder Rahmen werden textintern, d. h. auf der Mikroebene der sprachlichen Formulierungen, durch partiell vorgefertigte Äußerungsstrukturen nach dem Prinzip der Formulierungsadäquatheit ,aufgefüllt“. Viele davon sind konventionell geprägte polylexikalische Einheiten (Heinemann und Heinemann 2002: 130f.). Adamzik (1995: 28) spricht in diesem Zusammenhang von „kommunikativen Routinen“; Heinemann (2000a: 516) von „Stilmustern“ oder „Text-Teilmustern“; Heinemann (2000b: 356-369) und Gülich und Hausendorf (2000: 369385) von „Vertextungsmustern“. Mikrostrukturelle vorgefertigte Äußerungsstrukturen sind aber genauso an typisierte Situationen des sozialen Handels gebunden. Seit der pragmatischen Wende ist dies mehrfach bewiesen worden, so z. B. bereits von Coulmas (1979: 239). Der Gedanke findet sich unter dem Stichwort „Regelmäßigkeiten im Sprachgebrauch“ (Busse 2005: 35 ) in der modernen Diskurslinguistik wieder und wird dort durch den sozial-kooperativen Charakter des sprachlichen Handels begründet.

Es ist offensichtlich, dass globale Textmuster nur in ihrem Grundgerüst statisch sein können. In realen Kommunikationssituationen werden Texte nicht (nur) nach Konventionen und Mustern systematisch abgearbeitet, sondern individuell nach Umfang und Qualität ausgestaltet (Antos 1982). Gerade das „Auffüllen“ der globalen Textmuster auf der Mikroebene der oberflächlichen Teilmuster, d. h. der konkreten sprachlichen Äußerungen, lässt Freiraum für Modifikation, Variation und (sprach)historische Wandelprozesse. Variation auf der Ebene der sprachlichen Mittel ist somit natürlich und ausgeprägt, sie affiziert allerdings das konventionalisierte globale Textmuster im Normalfall nicht. Als ein Beispiel mögen hier die Luxemburger Rechnungsbücher aus dem 13.-17. Jahrhundert dienen, die in der gesamten Überlieferung keine kontinuierliche lineare oder fortschreitende Entwicklung zu einem endgültigen festen „Formelinventar" zeigen, sondern ein dauerhaftes Nebeneinander von einer festen modellhaften Grundstruktur und wechselnden Erscheinungen auf der Ebene der konkreten Formulierungen (Filatkina 2011: 93).

Ähnliches gilt selbst für die Anfänge der schriftlichen Überlieferung in deutscher Sprache. Den frühsten althochdeutschen und altsächsischen Beichten aus dem 9.-11. Jahrhundert hat bereits die ältere Forschung ein hohes Maß an Formelhaftigkeit sowohl im Textaufbau als auch auf der Ebene des konkreten Ausdrucks attestiert (Baeseke 1925: 279; Eggers 1955: 103). Oft wurde auf Ähnlichkeiten zwischen den zwölf überlieferten Beichten hingewiesen, die zur Annahme eines Verwandtschaftsverhältnisses geführt haben. Dadurch wurde auch das Vorhandensein ähnlicher fester Formulierungen erklärt und das Ziel formuliert, diesen den deutschensprachigen Beichttexten zugrunde liegenden Archetyp stemmatologisch zu rekonstruieren. Was dabei übersehen wurde, ist die gleichzeitige erhebliche Variation auf beiden Ebenen, die mit verfestigten Mustern ko-existiert und sich nicht als Abweichung vom Archetyp abtun lässt. Deshalb geht die neuere Forschung (Bulitta 2006: 50; Hellgardt 2013: 38-39; Gottwald und Hanauska 2013) vielmehr davon aus, dass die Texte unabhängig voneinander entstanden sind, ihre Verfasser/Schreiber aber auf ein zur damaligen Zeit existierendes Metawissen über die Textsorte Beichte, auf eine möglicherweise vom Lateinischen beeinflusste Formulierungstradition zurückgreifen. Dies erklärt das Vorhandensein eines viergliedrigen Aufbauschemas mit der einleitenden Bekenntnisformel, der Aufzählung der Sünden, dem Versprechen der Besserung und der Bitte um die Sündenvergebung, das nicht in allen Texten prototypisch umgesetzt, aber meist

ISSN 1615-3014 
erkennbar ist und das insbesondere auf der Ebene des konkreten sprachlichen Ausdrucks unterschiedlich ausfallen kann. Gottwald und Hanauska (2013: 456-472) analysieren dieses Oszillieren zwischen Festigkeit und Variation anhand des Beichten-Teilkorpus der HiFoS-Gruppe und weisen es für die einleitende Adressierungsformel (10), die Pflichtformel (11) und die Universalformeln wie in (12) nach:

(10) Truhtin dir uuirdu ich pigih|tik allero minero suntiono enti missetatio alles des ich | io missasprah [...] (Altbairische Beichte, 328, 1; ID 4750)

[Herr, dir bekenne ich alle meine Sünden und Missetaten, alles, was ich jemals falsch sagte oder falsch tat oder falsch dachte, Worte und Taten und Gedanken.]

(11) (...) gihorsam ni uuas so ih solta (Mainzer Beichte, 33v, 10; ID 32274)

[(...) ich war nicht so gehorsam, wie ich sollte]

(12) Ih gihu unrehtero gisihto . unrehtera gihorida | unrehtero gidanco . unrehdero uuordo . unrehdero uuerco (...) (Lorscher Beichte 3r, 21; ID 13124)

[Ich bekenne, [dass ich] Unrechtes sah, Unrechtes gehört [habe], unrechte Gedanken, unrechte Worte, unrechte Werke (...).]

Sie kommen zum folgenden Schluss:

Hierbei mag mit Sicherheit die ältere Tradition der lateinischsprachigen Beichtliteratur und die überregionale Verbreitung lateinischer Beichtformulare einen nicht zu unterschätzenden Einfluss auf die Ausbildung der deutschsprachigen Beichttexte gehabt haben. Andererseits deuten sich für bestimmte Einzelphänomene doch immer wieder eigene Formulierungstraditionen an, die zwar durchaus auf einer inhaltlichen Ebene gemeinsame Wurzeln haben, aber doch in struktureller, syntaktischer, lexikalischer und/oder morphosyntaktischer Hinsicht eine gewissen Eigenständigkeit beweisen.

(Gottwald und Hanauska 2013: 473)

Versuchen wir diese Beispiele konstruktionsgrammatisch zu erklären. Sprachsoziologie, Kontextualisierungstheorie, Formulierungs- und Textproduktionsforschung sowie zuletzt Text- und Diskurslinguistik zeigen, dass sowohl die innere Textstruktur als auch die sprachliche Oberfläche eines Textes an konventionalisierte Wissensstrukturen über diese Texte bzw. Textsorten gekoppelt sind. Das gilt für die Synchronie genauso wie für die Diachronie, für die Epochen der frühen, erst im Entstehen begriffenen Schriftlichkeit. Abstrakt gedacht ist diese Rückkopplung mit der konventionalisierten Form-/Bedeutungspaarung vergleichbar; sie ist aber mit der letzteren nicht identisch. Die bereits klassisch gewordene These Goldbergs (2003: 223) „It's constructions all the way down!“ wäre nach oben um die größere Dimension des Textes bzw. der Textsorte zu erweitern, in der das gesellschaftliche Metawissen über eine bestimmte Formulierungstradition - und nicht einfach der Kontext - gleichzeitig für die formelhafte Geprägtheit und Variation sorgt.

\section{Fazit}

In diesem Beitrag wurden die Wechselwirkungen zwischen der Konstruktionsgrammatik und der Formelhafte-Sprache-Forschung aus diachroner Perspektive diskutiert. Es zeigt sich, dass solche Auswirkungen sogar bei Wendungen möglich und nötig sind, die synchron betrachtet 
stark spezifisch bzw. lexikalisiert sind und in der Sprachgeschichte über ein hohes Variationspotential verfügen. Von der Variation und dem Wandel sind nicht nur die Form und die Bedeutung der Wendungen betroffen, sondern viele andere Ebenen auch. Dank der Betrachtung der Sprache als Ganzes (language system in its entirety) und der Orientierung an ebenenübergreifenden, nicht systemischen Veränderungen liefert die Konstruktionsgrammatik für die Beschreibung solcher Prozesse einen guten, aber auch einen ausbaufähigen theoretischen Rahmen. Wie die Analysen zeigen, können die Veränderungen einerseits simultan stattfinden, andererseits zieht die Veränderung auf einer Ebene nicht unbedingt die Veränderung auf der anderen nach sich. In den allermeisten Fällen sind die Veränderungen kleinschrittig und haben ihren Anfang auf der Ebene der konkreten Belege (utterances, Mikrokonstruktionen), nicht der der (Makro-)Konstruktionen. In der Nachzeichnung dieser Konventionalisierungsprozesse, der detaillierten Beschreibung der kleinschrittigen Dynamik und nicht unbedingt in erster Linie in der Ableitung der abstrakten Generalisierungen soll das Ziel der Erforschung der historischen formelhaften Sprache im konstruktionsgrammatischen Paradigma bestehen. Dabei kann die diachron orientierte Konstruktionsgrammatik vom begrifflichen Apparat der historischen Phraseologieforschung, der Sprachgeschichte und der Sprachwandelforschung profitieren, indem sie sich nicht mehr schwerpunkmäßig auf die Entstehung und Entwicklung abstrakter produktiver grammatischer Strukturen beschränkt, sondern sich auch anderen Phänomenen (z. B. formelhaften Wendungen) öffnet und weitere Faktoren des Sprachwandels in die Diskussion miteinbezieht.

\section{Primärliteratur}

Datenbank der Forschergruppe „Historische formelhafte Sprache und Traditionen des Formulierens (HiFoS)“: http://www.hifos.uni-trier.de [21.12.2017].

\section{Literatur}

Adamzik, Kirsten (1995): „Aspekte und Perspektiven der Textsortenlinguistik“. In: Adamzik, Kirsten (ed.): Textsorten - Texttypologie. Eine kommentierte Bibliographie. Münster, Nodus: $11-40$.

Antos, Gerd (1982): Grundlagen einer Theorie des Formulierens. Textherstellung in geschriebener und gesprochener Sprache. Tübingen: Niemeyer.

Antos, Gerd (1989): „Textproduktion. Ein einführender Überblick“. In: Antos, Gerd/Krings, Hans P. (eds.): Textproduktion. Tübingen, Niemeyer: 5-57.

Aurich, Claudia (2012): Proverb structure in the history of English: Stability and change. A corpus-based Study. Baltmannsweiler: Hohengehren.

Aurich, Claudia (2013): "Structural proverb change in English: The role of convergence processes”. In: Benayoun, Jean-Michel/Kübler, Natalie/Zouogbo, Jean-Philippe (eds.): Parémiologie. Proverbes et formes voisines. France, Presses Universitaires de Sainte Gemme: 123-132.

$A W B=$ Althochdeutsches Wörterbuch . Auf Grund der von Elias von Steinmeyer hinterlassenen Sammlungen im Auftrag der Sächsischen Akademie der Wissenschaften zu Leipzig begründet von Elisabeth Karg-Gasterstädt und Theodor Frings, hg. v. Gotthard Lerchner und Hans Ulrich Schmid unter der Leitung von Ingeborg Köppe und Brigitte Bulitta. Berlin 1968ff. 
Baesecke, Georg (1925): „Die Altdeutschen Beichten“. Beiträge zur Geschichte der deutschen Sprache und Literatur 49: 268-355.

Barđdal, Jóhanna (2013): "Construction-based historical-comparative reconstruction". In: Hoffmann, Thomas/Trousdale, Graeme (eds.): The Oxford Handbook of Construction Grammar. Oxford/New York, Oxford University Press: 438-457.

Berger, Peter/Luckmann, Thomas (1966/2010): Die gesellschaftliche Konstruktion der Wirklichkeit. Eine Theorie der Wissenssoziologie. 23. Auflage. Frankfurt a. M.: Fischer [Orig. engl. The social construction of reality. New York 1960].

Bergs, Alexander/Diewald, Gabriele (2008): "Introduction: Constructions and language change". In: Bergs, Alexander/Diewald, Gabriele (eds.): Constructions and language change. Berlin/New York, de Gruyter: 1-21.

Besch, Werner (1998): „... sein Licht (nicht) unter den Scheffel stellen“. In: Ernst, Peter/Patocka, Franz (eds.): Deutsche Sprache in Raum und Zeit. Festschrift für Peter Wiesinger zum 60. Geburtstag. Wien, Praesens: 463-477.

Besch, Werner (1999): „Wider den Stachel löcken/lecken“. In: Carr, Gerald/Harbert, Wayne/Zhang, Lihua (eds.): Interdigitations: Essays for Irmengard Rauch. New York u. a., Peter Lang: 247-256.

Bierich, Alexander (2005): Das phraseologische System der russischen Sprache des 18. Jahrhunderts. Entstehung, Semantik, Entwicklung. Frankfurt a. M.: Peter Lang.

Birkner, Karin (2007): „,Was X betrifft‘. Textsortenspezifische Aspekte einer Redewendung““. In: Stefanowitsch, Anatol/Fischer, Kerstin (eds.): Konstruktionsgrammatik II. Von der Konstruktion zur Grammatik. Tübingen, Niemeyer: 59-80.

Bulitta, Brigitte (2006): „Ein verkanntes althochdeutsches Sprachdenkmal: Die latenisch-deutsche Beichte von Zeitz“. In: Deye, Detlef/Rittig, Roland (eds.): Die Stiftsbibliothek und das Stiftsarchiv Zeitz. Halle/Saale, Schriften des Museums Schloss Moritzburg Zeitz: 47-74.

Burger, Harald (1987): „Normative Aspekte der Phraseologie“. In: Korhonen, Jarmo (ed.): Beiträge zur allgemeinen und germanistischen Phraseologieforschung. Oulu, Germanistisches Institut: $65-89$.

Burger, Harald (2012): „Alte und neue Fragen, alte und neue Methoden der historischen Phraseologie“. In: Filatkina, Natalia et al. (eds.): Aspekte der historischen Phraseologie und Phraseographie. Heidelberg, Winter: 1-20.

Burger, Harald/Linke, Angelika ( $\left.{ }^{2} 2008\right)$ : „Historische Phraseologie“. In: Besch, Werner/Betten, Anne/ Reichmann, Oskar et al. (eds.): Sprachgeschichte. Ein Handbuch zur Geschichte der deutschen Sprache und ihrer Erforschung. 2., vollständig neu bearbeitete und erweiterte Auflage. Band 1. Berlin/New York, de Gruyter: 743-755.

Buridant, Claude (2007): „Historische Phraseologie des Französischen“. In: Burger, Harald/Dobrovol'skij, Dmitrij/Kühn, Peter et al. (eds.): Phraseologie. Ein internationales Handbuch der zeitgenössischen Forschung. Halbband 2. Berlin/New York, de Gruyter: 1106-1125.

Busse, Dietrich (2005): „Sprachwissenschaft als Sozialwissenschaft?“. In: Busse, Dietrich/Niehr, Thomas/Wengeler, Martin (eds.): Brisante Semantik. Neuere Konzepte und Forschungsergebnisse einer kulturwissenschaftlichen Linguistik. Tübingen, Niemeyer: 21-43.

Bybee, Joan (2002): "Sequentiality as the basis of constituent structure". In: Givón, Talmy/Malle, Bertram F. (eds.): The evolution of language out of pre-language. Amsterdam/Philadelphia, John Benjamins: 109-132. 
Bybee, Joan (2010): Language, usage and cognition. Cambridge: Cambridge University Press. Coulmas, Florian (1979): "On the sociolinguistic relevance of routine formulae". Journal of pragmatics 3: 239-266.

Croft, William (2001): Radical construction grammar. Syntactic theory in typological perspective. Oxford: Oxford University Press.

Diewald, Gabriele ( $\left.{ }^{2} 2008 \mathrm{a}\right)$ : „Konstruktionen in der diachronen Sprachwissenschaft“. In: Fischer, Kerstin/Stefanowitsch, Anatol (eds.): Konstruktionsgrammatik: Von der Anwendung zur Theorie. 2. Auflage. Tübingen, Stauffenburg: 79-103.

Diewald, Gabriele (2008b): „Die Funktion ,idiomatischer' Konstruktionen bei Grammatikalisierungsprozessen - illustriert am Beispiel der Modalpartikel ruhig“. In: Stefanowitsch, Anatol/Fischer, Kerstin (eds.): Konstruktionsgrammatik II. Von der Konstruktion zur Grammatik. Tübingen, Stauffenburg: 33-57.

Diewald, Gabriele/Habermann, Mechthild (2005): „Die Entwicklung von werden + Infinitiv als Futurgrammem. Ein Beispiel für das Zusammenwirken von Grammatikalisierung, Sprachkontakt und soziokulturellen Faktoren“. In: Leuschner, Torsten/Mortelmans, Tanja/De Groodt, Sarah (eds.): Grammatikalisierung im Deutschen. Berlin/New York, de Gruyter: 229-250.

van Dijk, Teun A. (1980): Textwissenschaft. Eine interdisziplinäre Einführung. Tübingen: Niemeyer.

Dobrovol'skij, Dmitrij (2010): „Deiktische Konstruktionen des Deutschen aus lexikographischer Perspektive“. In: Dykstra, Anne/Schoonheim, Tanneke (eds.): Proceedings of the XIV Euralex International Congress, Leeuwarden, 6-10 July 2010. CD-ROM. Leeuwarden, Fryske Akademy: 1282-1290.

Dobrovol'skij, Dmitrij (2011): „Phraseologie und Konstruktionsgrammatik“. In: Lasch, Alexander/Ziem, Alexander (eds.): Konstruktionsgrammatik III. Aktuelle Fragen und Lösungsansätze. Tübingen, Stauffenburg: 111-130.

Dobrovol'skij, Dmitrij/Piirainen, Elisabeth (2009): Zur Theorie der Phraseologie. Kognitive und kulturelle Aspekte. Tübingen: Narr.

Dobrovol'skij, Dmitrij/Šarandin, Artëm (2010): „Konstruktionsgrammatik und Lexikographie: Verben der Fortbewegung im Neuen deutsch-russischen Großwörterbuch“. In: Durco, Peter (ed.): Feste Wortverbindungen und Lexikographie. Kolloquium zur Lexikographie und Wörterbuchforschung. Berlin/Boston, de Gruyter: 37-41.

Dräger, Marcel (2011): Der phraseologische Wandel und seine lexikographische Erfassung. Konzept des ,,Online-Lexikons zur diachronen Phraseologie (OldPhras) “. Basel: Universität Basel.

Eggers, Hans (1955): „Die altdeutschen Beichten I“. Beiträge zur Geschichte der deutschen Sprache und Literatur 77: 89-123.

Eisenberg, Peter ( $\left.{ }^{2} 1989\right):$ Grundriss der deutschen Grammatik. 2. Auflage. Stuttgart: Metzler.

Feilke, Helmuth (1994): Commen sence-Kompetenz. Überlegungen zu einer Theorie ,sympatischen “ und „,natürlichen "Meinens und Verstehens. Frankfurt a. M.: Suhrkamp.

Feilke, Helmuth (2004): „Kontext - Zeichen - Kompetenz. Wortverbindungen unter sprachtheoretischem Aspekt"،. In: Steyer, Kathrin (ed.): Wortverbindungen - mehr oder weniger fest. Berlin/New York, de Gruyter: 41-64.

Feilke, Helmuth (2007): „Syntaktische Aspekte der Phraseologie III: Construction Grammar und verwandte Ansätze“. In: Burger, Harald/Dobrovol'skij, Dmitrij/Kühn, Peter et al. (eds.): 
Phraseologie. Ein internationales Handbuch der zeitgenössischen Forschung. Halbband 1. Berlin/New York, de Gruyter: 63-76.

Feilke, Helmuth (2012): „Was sind Textroutinen? - Zur Theorie und Methodik des Forschungsfeldes“. In: Feilke, Helmuth/Lehnen, Katrin (eds.): Schreib- und Textroutinen. Theorie, Erwerb und didaktisch-mediale Modellierung. Frankfurt a. M., Peter Lang: 1-31.

Filatkina, Natalia (2005): Phraseologie des Lëtzebuergeschen. Strukturelle, semantisch-pragmatische und bildliche Aspekte. Heidelberg: Winter.

Filatkina, Natalia (2011): „Variation im Bereich der formelhaften Wendungen am Beispiel der Luxemburger Rechnungsbücher (1388-1500)“. In: Elspaß, Stephan/Negele, Michaela (eds.): Sprachvariation und Sprachwandel in der Stadt der Frühen Neuzeit. Heidelberg, Winter: 79-95.

Filatkina, Natalia (2012): “Wan wer beschreibt der welte stat / der muoß wol sagen wie es gat. Manifestation, functions and dynamics of formulaic patterns in Thomas Murner's ,Schelmenzunft' revisited". In: Filatkina, Natalia et al. (eds.): Aspekte der historischen Phraseologie und Phraseographie. Heidelberg, Winter: 21-44.

Filatkina, Natalia (2013): „Wandel im Bereich der historischen formelhaften Sprache und seine Reflexe im Neuhochdeutschen: Eine neue Perspektive für moderne Sprachwandeltheorien“. In: Vogel, Petra (ed.): Sprachwandel im Neuhochdeutschen. Jahrbuch der Gesellschaft für germanistische Sprachgeschichte 2013. Berlin/New York, de Gruyter: 34-51.

Filatkina, Natalia (2015): „Constructionalization, Konstruktionswandel und figurative Sprache (sprach)historisch betrachtet“. In: Dalmas, Martine/Piirainen, Elisabeth (eds.): Figurative Sprache. Festschrift für Dmitrij Dobrovol'skij zum 60. Geburtstag. Unter Mitarbeit von Natalia Filatkina. Tübingen, Stauffenburg: 41-58.

Filatkina, Natalia (im Druck): „Wie fest sind feste Strukturen? Beobachtungen zu Varianz in (historischen) Wörterbüchern und Texten“. In: Borek, Luise/Rapp, Andrea/Wegstein, Werner (eds.): Vielfalt und Varianz interdisziplinär. Mannheim: Institut für deutsche Sprache.

Filatkina, Natalia (2018): Historische formelhafte Sprache. Theoretische Grundlagen und Methoden ihrer Erforschung. Berlin/Boston: de Gruyter.

Fillmore, Charles J. (1976): "Pragmatics and the description of discourse". In: Schmidt, Siegfried (ed.): Pragmatik/Pragmatics II. Grundlegung einer expliziten Pragmatik. München, Fink: 83-104.

Fried, Mirjam (2009a): "Representing contextual factors in language change: between frames and constructions". In: Bergs, Alexander/Diewald, Gabriele (eds.): Contexts and constructions. Amsterdam/Philadelphia, John Benjamins: 63-94.

Fried, Mirjam (2009b): "Construction grammar as a tool for diachronic analysis". In: Constructions and Frames 1/2: 262-291.

Fried, Mirjam (2013): "Principles of constructional change". In: Hoffmann, Thomas/Trousdale, Graeme (eds.): The Oxford Handbook of Construction Grammar. Oxford/New York, Oxford University Press: 419-437.

Friedrich, Jesko (2007): „Historische Phraseologie des Deutschen“. In: Burger, Harald/Dobrovol'skij, Dmitrij/Kühn, Peter et al. (eds.): Phraseologie. Ein internationales Handbuch der zeitgenössischen Forschung. Halbband 2. Berlin/New York, de Gruyter: 1092-1106. (= HSK).

Goldberg, Adele E. (1995): Constructions. A construction grammar approach to argument structure. Chicago/London: The University of Chicago Press. 
Goldberg, Adele (2003): "Constructions: a new theoretical approach to language". Trends in Cognitive Sciences 7/5: 219-224.

Gottwald, Johannes/Hanauska, Monika (2013): „Formelhafte Sprache in den althochdeutschen und altsächsischen Beichten“. Sprachwissenschaft 38/4, 445-476.

Gülich, Elisabeth/Hausendorf, Heiko (2000): „Vertextungsmuster Narration“. In: Brinker, Klaus/Antos, Gerd/Heinemann, Wolfgang et al. (eds.): Text- und Gesprächslinguistik. Ein internationales Handbuch zeitgenössischer Forschung. Band 1. Berlin/New York, de Gruyter: 369-385. (=HSK).

Gumperz, John (1982): Discourse strategies. Cambridge: Cambridge University Press.

Gvozdarev, Jurij (1981): „Phrasenbildende Prozesse und damit verbundene Begriffe“. In: Jaschke, Harald/Sialm, Ambros/Burger, Harald (eds.): Reader zur sowjetischen Phraseologie. Berlin/New York, de Gruyter: 113-129.

Hanauska, Monika (2014): ,Historia dye ist ein gezuyge der zijt ... “. Untersuchungen zur pragmatischen Formelhaftigkeit in der volkssprachigen Kölner Stadthistoriographie des Spätmittelalters. Heidelberg: Winter.

Heinemann, Margot/Heinemann, Wolfgang (2002): Grundlagen der Textlinguistik. Interaktion - Text-Diskurs. Tübingen: Niemeyer.

Heinemann, Wolfgang (2000a): „Textsorte - Textmuster - Texttyp“. In: Brinker, Klaus/Antos, Gerd/Heinemann, Wolfgang et al. (eds.): Text- und Gesprächslinguistik. Ein internationales Handbuch zeitgenössischer Forschung. Band 1. Berlin/New York, de Gruyter: 507-523. (= HSK).

Heinemann, Wolfgang (2000b): „Vertextungsmuster Deskription“. In: Brinker, Klaus/Antos, Gerd/Heinemann, Wolfgang et al. (eds.): Text- und Gesprächslinguistik. Ein internationales Handbuch zeitgenössischer Forschung. Band 1. Berlin/New York, de Gruyter: 356-369. (= HSK).

Hellgardt, Ernst (2013): „Beichten, althochdeutsche und altsächsische“. In: Bergmann, Rolf (ed.): Althochdeutsche und altsächsische Literatur. Berlin/Boston, de Gruyter: 38-46.

Hilpert, Martin (2011): „Was ist Konstruktionswandel?““. In: Lasch, Alexander/Ziem, Alexander (eds.): Konstruktionsgrammatik III. Aktuelle Fragen und Lösungsansätze. Tübingen, Stauffenburg: 59-75.

Hilpert, Martin (2013): “Corpus-based approaches to constructional change". In: Hoffmann, Thomas/Trousdale, Graeme (eds.): The Oxford Handbook of Construction Grammar. Oxford/New York, Oxford University Press: 458-475.

Hilpert, Martin (2014): Construction grammar and its application to English. Edinburgh: Edinburgh University Press.

Hoff, Carina (2012): „Si sagent vns guode wort. Formelhafte Sprache in den südwestdeutschen Nonnenviten des 14. Jahrhunderts“. In: Filatkina, Natalia/Kleine-Engel, Ane/Dräger, Marcel/Burger, Harald (eds.): Aspekte der historischen Phraseologie und Phraseographie. Heidelberg, Winter: 67-82.

Hüpper, Dagmar/Topalovic, Elvira/Elspaß, Stephan (2002): „Zur Entstehung und Entwicklung von Paarformeln im Deutschen“. In: Piirainen, Elisabeth/Piirainen, Ilpo Tapani (eds.): Phraseologie in Raum und Zeit. Akten der 10. Tagung des Westfälischen Arbeitskreises „Phraseologie/Parömiologie " (Münster 2001). Baltmannsweiler, Hohengehren: 77-99.

Kay, Paul/Fillmore, Charles J. (1999): „Grammatical constructions and linguistic generalizations: The What's X doing Y? construction“. Language 75/1: 1-33.

ISSN 1615-3014 
Kleine-Engel, Ane (2011): ,fannen as wannen. Das Phraseologiemodul in LexicoLux“. In: Gilles, Peter/Wagner, Melanie (eds.): Aktuelle Forschungsfragen in der Luxemburgistik. Frankfurt a. M., Lang: 271-292.

Kleine-Engel, Ane (2012): "Some arguments for a historical approach to phraseology in not (fully) standardized languages". In: Filatkina, Natalia et al. (eds.): Aspekte der historischen Phraseologie und Phraseographie. Heidelberg, Winter: 127-145.

Kohrt, Manfred ( $\left.{ }^{2} 1998\right)$ : „Historische Phonologie und Graphematik“. In: Besch, Werner et al. (eds.): Sprachgeschichte. Ein Handbuch zur Geschichte der deutschen Sprache und ihrer Erforschung. 2., vollständig neu bearbeitete und erweiterte Auflage. Band 1. Berlin/New York, de Gruyter: 551-572. (= HSK).

Kuusi, Matti (1974): „Tiefenstruktur und Oberflächenstruktur in der Parömiologie“. Proverbium 23: 920-928.

Langacker, Ronald W. (1977): “Syntactic reanalysis". In: Li, Charles (ed.): Mechanisms of syntactic change. Austin, University of Texas: 57-139.

Lehmann, Christian (1989): „Grammatikalisierung und Lexikalisierung“. Zeitschrift für Phonetik, Sprachwissenschaft und Kommunikationsforschung 42: 11-19.

Lehmann, Christian (1995): Thoughts on Grammaticalization. München: Lincom Europa.

Luckmann, Thomas (1986): „Grundformen der gesellschaftlichen Vermittlung des Wissens: Kommunikative Gattungen“. In: Neidhardt, Friedhelm/König, René (eds.): Kultur und Gesellschaft. Opladen, Westdeutscher Verlag: 191-211.

Mattheier, Klaus ( $\left.{ }^{2} 2000\right)$ : „Die Herausbildung neuzeitlicher Schriftsprachen“. In: Besch, Werner et al. (eds.): Sprachgeschichte. Ein Handbuch zur Geschichte der deutschen Sprache und ihrer Erforschung. 2., vollständig neu bearbeitete und erweiterte Auflage, Band 2. Berlin/New York, de Gruyter: 1085-1107. (=HSK).

Mieder, Wolfgang (2009): International bibliography of paremiology and phraseology. 2 volumes. Berlin/New York: de Gruyter.

Mokienko, Valerij (2002): „Prinzipien einer historisch-etymologischen Analyse der Phraseologie“. In: Hartmann, Dietrich/Wirrer, Jan (eds.): Wer A sägt, muss auch B sägen. Beiträge zur Phraseologie und Sprichwortforschung aus dem Westfälischen Arbeitskreis. Baltmannsweiler, Hohengehren: 231-254.

Noël, Dirk (2007): „Diachronic construction grammar and grammaticalization theory“. Functions of Language 14/2: 177-202.

Östman, Jan-Ola/Trousdale, Graeme (2013): "Dialects, discourse, and construction grammar". In: Hoffmann, Thomas/Trousdale, Graeme (eds.): The Oxford Handbook of Construction Grammar. Oxford/New York, Oxford University Press: 476-490.

Permjakov, Grigorij L. (1979/1984): «Грамматика пословичной мудрости». In: Пословищьь и поговорки народов востока. Москва, 7-57. In deutscher Übersetzung: „Die Grammatik der Sprichwörterweisheit“. In: Grzybek, Peter (ed.): Semiotische Studien zum Sprichwort. Simple forms reconsidered I. Unter Mitarbeit von Wolfgang Eismann. Tübingen, Narr: 295344.

von Polenz, Peter (1963): Funktionsverben im heutigen Deutsch. Sprache in der rationalisierten Welt. Düsseldorf: Schwann. (= Beihefte zur Zeitschrift „Wirkendes Wort“ 5).

Piirainen, Elisabeth (2012): Widespread idioms in Europe and beyond. Toward a lexicon of common figurative units. New York etc.: Peter Lang.

Röhrich, Lutz/Mieder, Wolfgang (1977): Sprichwort. Stuttgart: Metzler. 
Rothkegel, Annelies (1989): Polylexikalität. Verb-Nomen-Verbindungen und ihre Behandlung. Saarbrücken: IAI. (= eurotra . iai eurotra-D Working papers 17)

Schowe, Ulrike (1994): „, Mit Haut und Haar“. Idiomatisierungsprozesse bei sprichwörtlichen Redensarten aus dem mittelalterlichen Strafrecht. Frankfurt a. M.: Peter Lang.

Schützeichel, Rudolf ( $\left.{ }^{6} 2006\right)$ : Althochdeutsches Wörterbuch. 6. Auflage, überarbeitet und um die Glossen erweitert. Tübingen: Niemeyer.

Sialm, Ambros/Burger, Harald/Linke, Angelika (1982): „Historische Phraseologie“. In: Burger, Harald/Buhofer, Annelies/Sialm, Ambros (eds.): Handbuch der Phraseologie. Berlin/New York, de Gruyter: 323-330.

Steyer, Kathrin (2012): „Sprichwortstatus, Frequenz, Musterbildung. Parömiologische Fragen im Lichte korpusmethodischer Empirie“. In: Steyer, Kathrin (ed.): Sprichwörter multilingual. Theoretische, empirische und angewandte Aspekte der modernen Parömiologie. Tübingen, Narr: 287-314.

Steyer, Kathrin (2013): Usuelle Wortverbindungen. Zentrale Muster des Sprachgebrauchs aus korpusanalytischer Sicht. Tübingen: Narr.

Stumpf, Sören (2014): „Mit Fug und Recht - Neue Erkenntnisse zu phraseologisch gebundenen Formativen (PGF)“. Sprachwissenschaft 39/1: 85-114.

Talmy, Leonard (1985): "Lexicalization patterns: semantic structure in lexical forms". In: Shopen, Timothy (ed.): Language typology and syntactic description. Volume 3: Grammatical categories and the lexicon. Cambridge, Cambridge University Press: 57-149.

Traugott, Elizabeth Closs (2003): "Constructions in grammaticalization". In: Joseph, Brian /Janda, Richard (eds.): The Handbook of Historical Linguistics. Oxford, Oxford University Press: 624-647.

Traugott, Elisabeth Closs (2008): “The grammaticalization of NP of NP patterns". In: Bergs, Alexander/Diewald, Gabriele (eds.): Constructions and language change. Berlin/New York, de Gruyter: 23-45.

Smirnova, Elena (2011): „Zur diachronen Entwicklung deutscher Komplementsatz-Konstruktionen“. In: Lasch, Alexander/Ziem, Alexander (eds.): Konstruktionsgrammatik III. Aktuelle Fragen und Lösungsansätze. Tübingen, Stauffenburg: 77-93.

Warnke, Ingo (1999): „Diskursivität und Intertextualität als Parameter sprachlichen Wandels. Prolegomena einer funktionalen Sprachgeschichtsschreibung“. In: Warnke, Ingo (ed.): Schnittstelle Text/Diskurs. Frankfurt a. M., Peter Lang: 215-222.

Weber, Beatrix (2015): „the seid acte, statute or ordenaunce, or eny other made to the contrary, notwithsondyng. Zur Rolle der Faktoren ,Diskurstradition“ und ,Sprachkontakt" bei der Etablierung der notwithstanding-Konstruktion im Englischen“. In: Ziem, Alexander/Lasch, Alexander (eds.): Konstruktionsgrammatik IV. Konstruktionen als soziale Konventionen und kognitive Routinen. Tübingen, Stauffenburg: 225-244.

Werner, Otmar ( ${ }^{2}$ 1998): „Historische Morphologie“. In: Besch, Werner et al. (eds.): Sprachgeschichte. Ein Handbuch zur Geschichte der deutschen Sprache und ihrer Erforschung. 2., vollständig neu bearbeitete und erweiterte Auflage. Band 1. Berlin/New York, de Gruyter: 572-596. (= HSK).

Wolk, Christoph/Bresnan, Joan/Rosenbach, Anette et al. (2013): "Dative and genitive variability in Late Modern English: Exploring cross-constructional variation and change". Diachronica 30/3: 382-419. 
Wray, Alison (2009): "Identifying formulaic language: Persistent challenges and new opportunities”. In: Corrigan, Roberta et al. (eds.): Formulaic language. Volume 1. Distribution and historical change. Amsterdam/Philadelphia, John Benjamins: 27-51.

Wray, Alison/Perkins, Michael R. (2000): "The functions of formulaic language: An integrated model". Language and communication 20/1: 1-28.

Ziem, Alexander (2015): „Desiderata und Perspektiven einer Social Construction Grammar“. In: Ziem, Alexander/Lasch, Alexander (eds.): Konstruktionsgrammatik IV. Konstruktionen als soziale Konventionen und kognitive Routinen. Tübingen, Stauffenburg: 1-21. 